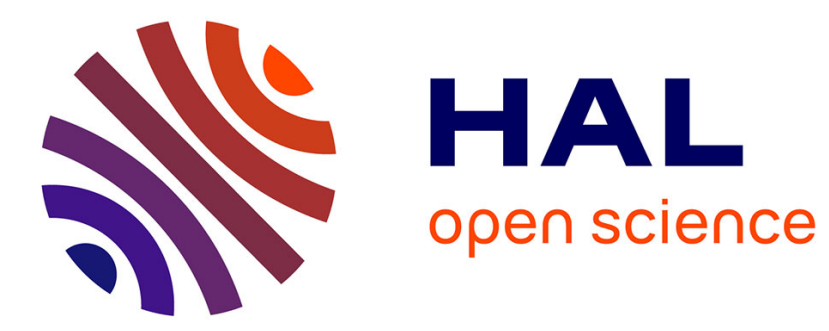

\title{
Mercure au Liban
}

Julien Aliquot

\section{- To cite this version:}

Julien Aliquot. Mercure au Liban. Topoi Orient - Occident, 2009, 16, pp.241-264. 10.3406/topoi.2009.2303 . halshs-00591260

\section{HAL Id: halshs-00591260 https://shs.hal.science/halshs-00591260}

Submitted on 29 Jan 2020

HAL is a multi-disciplinary open access archive for the deposit and dissemination of scientific research documents, whether they are published or not. The documents may come from teaching and research institutions in France or abroad, or from public or private research centers.
L'archive ouverte pluridisciplinaire HAL, est destinée au dépôt et à la diffusion de documents scientifiques de niveau recherche, publiés ou non, émanant des établissements d'enseignement et de recherche français ou étrangers, des laboratoires publics ou privés. 


\section{MERCURE AU LIBAN *}

$\mathrm{Au}$ Proche-Orient, les fidèles invoquent parfois Hermès sous son nom grec, mais ce dieu dont on ne connaît qu'un sanctuaire, à Antioche, conserve un caractère hellénique, soit comme patron de l'agora ou du gymnase, soit comme conducteur des âmes défuntes ${ }^{1}$. Un culte différent, celui de Mercure, est par ailleurs une spécialité libanaise bien connue ${ }^{2}$. Curieusement, en dépit de l'intérêt suscité par la triade divine d'Héliopolis-Baalbek, composée de Jupiter, Vénus et Mercure, ces faits sont restés inaperçus : même si l'on s'est avisé que, près de Baalbek, les dieux prenant le nom ou l'aspect de Mercure sont loin d'être tous assimilables à l'acolyte du Jupiter héliopolitain, on s'est efforcé de rapporter toutes ces divinités au type d'un dieu phénicien de la végétation assimilé au Soleil et à Bacchus ; et c'est ce dieu, comme on l'a supposé à la suite d'H. Seyrig, qui aurait été placé au centre d'un culte à mystères dont la vogue aurait justifié la construction du temple qui s'élève au sud du grand sanctuaire de Baalbek, avec le soutien de Rome et de nombreuses cités du Proche-Orient ${ }^{3}$. Tout en laissant à d'autres le soin d'apprécier

* Cet article a fait l'objet d'une communication au quatrième colloque international sur les Sanctuaires du Proche Orient hellénistique et romain, consacré aux Temples et sanctuaires du Liban et organisé à Beyrouth par P.-L. Gatier (CNRS, UMR 5189 HiSoMA, Lyon) et L. Nordiguian (Université Saint-Joseph, Beyrouth) les 26 et 27 avril 2007. Je remercie J.-C. Bessac (CNRS, Institut français du Proche Orient, Damas) et P.-L. Gatier d'avoir accepté de le relire. Les erreurs et les omissions qui pourraient subsister dans les pages qui suivent sont de mon entière responsabilité.

1. Voir infra, «Appendice : Hermès au Proche-Orient ».

2. La dédicace de Bostra IGLS 13/1, 9016 n'infirme pas ce constat, puisqu'elle émane d'expatriés originaires de Thysdrus (aujourd'hui el-Djem, en Tunisie) et non d'Orientaux : Mercu/rio Aug(usto) / sacrum,/ Thusdr(i)/tani / Gen(io) col(oniae) / s(uae) f(ecerunt), « les Thysdritains ont fait (faire) ce monument consacré à Mercure Auguste, au génie de leur colonie ». Le culte de Mercure est attesté à el-Djem par la dédicace CIL 8, 22845 : deo Mercurio, san(c)to Genio co[lo]niae Thysdrita[no]rum.

3. H. SeYrig, Syria 31 (1954), p. 81-88 (Antiquités syriennes 5, p. 100-107).

Topoi 16 (2009)

p. $241-264$ 
ce qu'un tel pot-pourri mythologique doit au Rameau d'Or de J. Frazer, je me propose de rouvrir le dossier libanais de Mercure pour préciser les caractéristiques du dieu de Baalbek et de ses alter ego, identifier leurs sanctuaires et revenir sur deux questions, celle de la formation du culte héliopolitain et celle des rapports entre Mercure, Sol et Bacchus.

\section{Le messager du Jupiter héliopolitain et ses alter ego}

Y. Hajjar réunit la majorité des textes et des monuments relatifs au Mercure héliopolitain dans ses travaux sur la triade divine d'Héliopolis-Baalbek ${ }^{4}$. Certes, on peut déplorer que cet auteur aggrave la tendance, déjà à l'œuvre chez ses prédécesseurs, consistant à annexer à son répertoire documentaire de nombreux témoignages dont la nature héliopolitaine est incertaine ${ }^{5}$. Néanmoins, si l'on utilise le matériau qu'il rassemble en tenant compte du fait que les dieux du Liban peuvent emprunter l'aspect des dieux de Baalbek, il devient possible de cerner l'identité du jeune parèdre de la triade. Pour ce faire, il paraît pertinent de ne prendre en considération que les textes et les monuments du culte public.

Le seul objet connu où le dieu soit nommé et représenté est un plomb votif découvert à Ain el-Jouj, à six kilomètres à l'est de Baalbek, dans l'un des bassins du sanctuaire $\left(\right.$ Fig. 1) ${ }^{6}$ : l'idole au pied de laquelle on lit la dédicace 'E $\rho \mu \tilde{n}$, « à Hermès », reproduit le type canonique du Jupiter héliopolitain, debout, de face, coiffé du calathos, le corps réduit à un terme et enserré dans une gaine ornée de motifs stellaires (disques, rosaces, étoiles) ${ }^{7}$. Toutefois, Mercure diffère du grand

4. Y. HaJjaR, La triade d'Héliopolis-Baalbek, Leyde (1977), en abrégé Triade 1-2. Corrections et suppléments : La triade d'Héliopolis-Baalbek, Montréal (1985), en abrégé Triade 3 ; LIMC 4 (1988), p. 573-592 ; ANRW 2, 18.4 (1990), p. 2458-2508.

5. J.-P. Rey-CoQuaIs, REA 82 (1980), p. 132-133, soulève à juste titre ce problème dans son compte rendu de Triade 1-2, sans que Y. Hajjar modifie son propos dans ses études ultérieures.

6. Il est rare qu'une dédicace assure l'identification des dieux de Baalbek, y compris pour le Jupiter héliopolitain, qui n'est reconnaissable par ce biais que sur quelques monuments provenant de régions très diverses : Triade 1-2, $\mathrm{n}^{\text {os }} 186$ (Palmyrène), 216-217 (Deir el-Qalaa), 250 (Chahba), 285 (Nîmes) ; L. BADRE, Syria 76 (1999), p. 185, fig. 4 (Ain el-Jouj). Aucun monument au type de la Vénus héliopolitaine ne porte le nom de la déesse.

7. IGLS 6, 2896 (Triade 1-2, $\mathrm{n}^{\circ}$ 117). Sur les plombs d'Ain el-Jouj, Triade 1-2, $\mathrm{n}^{\text {os }} 107128$; Triade 3, p. 348-349 ; L. BADRE, Syria 76 (1999), p. 181-196. La majorité de ces monuments représente le Jupiter héliopolitain. Mercure vient en seconde position, loin devant les autres personnages identifiables (Dionysos, Satyre, Héraclès, Tyché et un dieu solaire imberbe, radié et drapé). Il est représenté selon cinq types : nu avec deux protubérances sur la tête, probablement des ailes ; nu avec un grand calathos sur la tête ; vêtu du chiton ou de la tunique ; en dieu-terme ; debout 


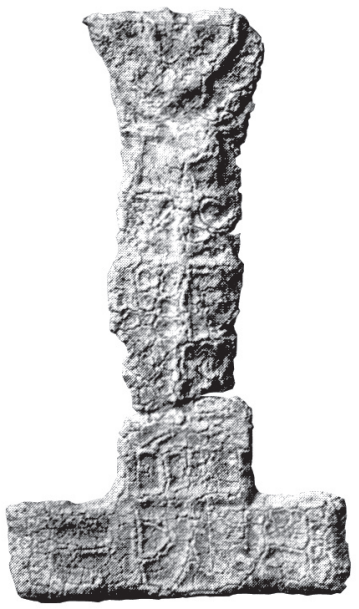

Fig. 1 - Plomb votif d'Ain el-Jouj, Staatliche Museen de Berlin. Photo Triade 1-2, $\mathrm{n}^{\circ} 117$, pl. 36 .
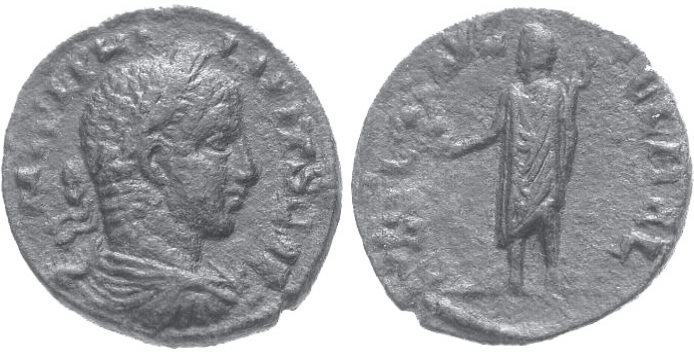

Fig. 2 - Monnaie de bronze d'Héliopolis : au droit, buste de Philippe, le fils de Philippe l'Arabe (244-249) ; au revers, Mercure debout de face, vêtu de la chlamyde, la tête de profil à gauche, la bourse à la main et le caducée sur l'épaule. Collection B. Peus, exemplaire publié le 28 avril 2004 sur le site CoinArchives.com, semblable à Triade 1-2, nº 94.

dieu en ce qu'il est flanqué de béliers et non de taureaux ${ }^{8}$. Il reste le protecteur des bergers et le passeur, même sur les monnaies d'Héliopolis, qui adoptent l'imagerie hermaïque grecque et romaine en le figurant la tête ailée, avec la bourse et le caducée, sans accorder aucune place au coq (Fig. 2) ${ }^{9}$. Enfin, bien qu'il soit la dernière divinité de la triade, Mercure porte le titre de dominus, à Baalbek comme à Béryte ${ }^{10}$, et reçoit même les attributs de la puissance cosmique sur l'autel inscrit de Btédaai : sur la face antérieure, il se drape dans un manteau et tient le caducée ; sur les faces latérales, le couple du Soleil et de la Lune le caractérise comme un dieu au pouvoir universel (Fig. 3) ${ }^{11}$.

sur une base, tenant peut-être le caducée à la main. Cinq exemplaires d'une même figurine le montrent flanqué de deux béliers. Lorsqu'il se présente nu ou vêtu de la chlamyde, il est muni du caducée.

8. H. SeYrig, Syria 31 (1954), p. 81-84 (Antiquités syriennes 5, p. 100-103), est le premier à mettre ce fait en évidence.

9. Triade 1-2, $\mathrm{n}^{\text {os }}$ 92-96 (sous Caracalla, Philippe l'Arabe, Valérien et Gallien).

10. Infra n. 32-34 (Baalbek); Triade 1-2, n ${ }^{\text {os }}$ 213-214 (Béryte).

11. L'autel est exposé au Musée national de Beyrouth (inv. 2607). Sa dédicace garantit son appartenance à la série des monuments héliopolitains. Elle semble encore imparfaitement établie. Voir H. SeYRIG, BMB 1 (1937), p. 91-95, fig. 4 (Scripta varia, p. 80-84) ; IGLS 6, 2910, pl. 43-44 (Triade 1-2, n 131), cf. AE 1939, 71

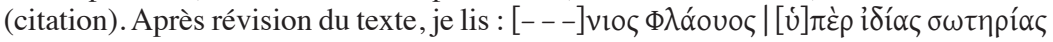



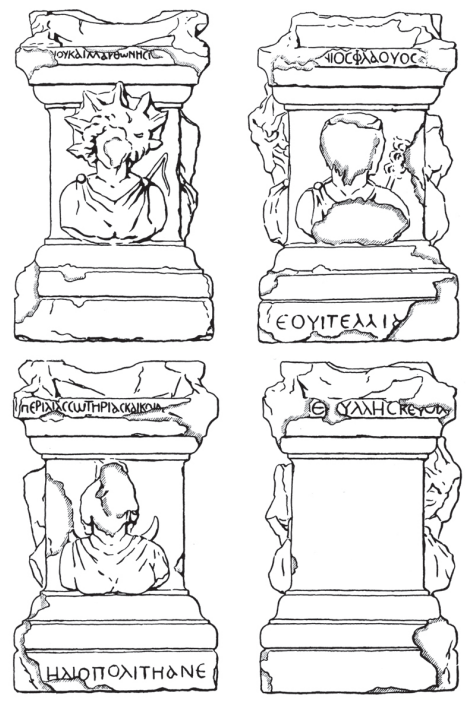

Fig. 3 - Autel de Btédaai, Musée national de Beyrouth. Facsimilé C. de la Chaussée, dans H. SeYrig, $B M B 1$ (1937), p. 93, fig. 4

(Scripta varia, p. 82).

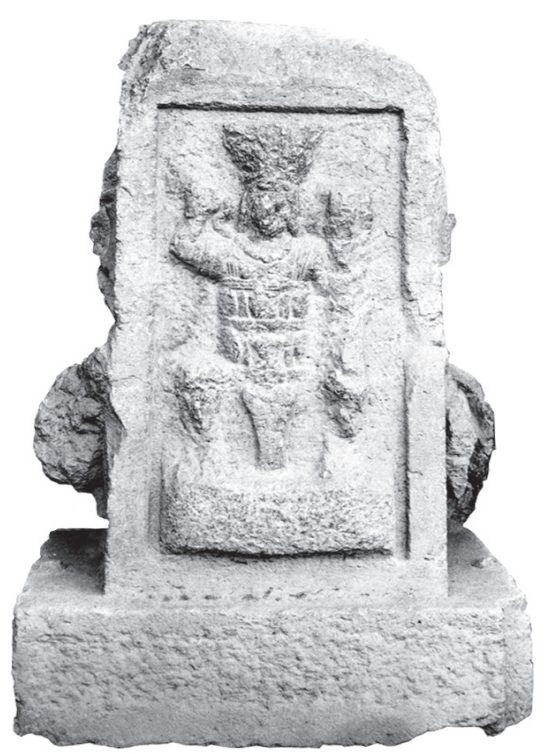

Fig. 4 - Monolithe d'Ain el-Jouj. Photo Triade 1-2, ${ }^{\circ}$ 108, pl. 30.

Le Mercure héliopolitain n'est jamais confondu avec Sol ou Bacchus. Sol n'est qu'un motif sur la gaine du dieu, comme sur celle de Jupiter ${ }^{12}$. Les médaillons de bronze de la Békaa, dont H. Seyrig a fait grand cas, associent bien Jupiter et Vénus à un buste solaire, mais non de manière systématique, tout comme les ex-voto d'Ain el-Jouj, qui présentent un assortiment de dieux et de héros ${ }^{13}$. Les monnaies d'Héliopolis où Y. Hajjar a cru reconnaître un Mercure solaire portent

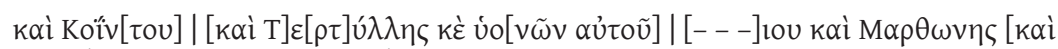

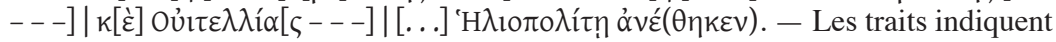
les sauts de lecture d'un côté à l'autre de la corniche, puis sur les deux faces de la base. - L. 1. H. Seyrig ne lit pas l'upsilon qui précède la lacune finale d'après $I G L S$

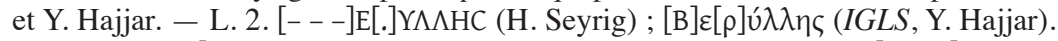

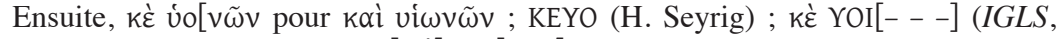
Y. Hajjar). - L. 6. Restituer $[\Delta i \mathrm{i}]$ ou $[\theta \varepsilon \tilde{\omega}]$. Traduction : « [...]-nius Flavus [...], pour son propre salut et pour celui de Quintus et Tertulla et pour celui de ses petitsenfants, [...]ios, Marthonè, [...] et Vitellia [...], a consacré (cet autel) à [...] (dieu) d'Héliopolis. »

12. Triade $1-2, \mathrm{n}^{\circ} 293$ (Rome).

13. H. SeYrig, Syria 48 (1971), p. 345-348, 367-370. 
en réalité l'image de Tyché avec une couronne stylisée ${ }^{14}$. Quant aux monuments dionysiaques du Liban, ils indiquent surtout la place remarquable de Bacchus aux côtés des dieux héliopolitains. À Baalbek, le temple au sud du grand sanctuaire est orné d'un riche décor bachique : sur le relief ornant le piédroit de la porte, une Ménade offre le sein à Bacchus en compagnie de Pan, d'Amours, de Satyres et d'autres Ménades ; sur les bas-reliefs de l'adyton, le dieu assiste d'un côté à la métamorphose de sa nourrice Ambrosia et de l'autre à la défaite de Lycurgue ${ }^{15}$. Sur l'autel de Béchouat, Mercure et Bacchus restent distincts, chacun sur une face ${ }^{16}$. Il faut donc s'en tenir aux deux types du Mercure héliopolitain : engainé et hermaïque.

Si l'on tient compte des monuments où l'on retrouve ses deux types canoniques, il semble que le dieu tienne une place particulière dans l'imagerie héliopolitaine, jouant le rôle d'un messager, comme dans la mythologie classique : sur le monolithe du sanctuaire d'Ain el-Jouj, son idole se dresse au pied de celle du grand dieu, en position d'hypostase (Fig. 4) ${ }^{17}$; Zeus d'Héliopolis est honoré à Qalaat Faqra « de la part d'Hermès » ${ }^{18}$; le caducée est aussi un attribut du grand dieu héliopolitain, sur le linteau de la porte du petit temple de Baalbek, dans les serres de l'aigle, et dans la cour du grand temple, sur l'autel monumental ${ }^{19}$. On pourrait retrouver Mercure en tant que héraut jovien dans l'épigramme de l'Anthologie palatine dont le lemme précise qu'il s'agit de l'« oracle rendu à

14. Triade $1-2, \mathrm{n}^{\text {os }} 100-101$ (Gallien), revenant à l'identification de Tyché dans LIMC 4 (1988), p. 583, nos 83-85. T. GANSCHOw, LIMC 8 (1997), p. 614, n 3b, reconnait lui aussi Tyché.

15. Baalbek 2, p. 36-37, 64-65, 81-85, pl. 51-52, avec les observations d'H. SeYRIG, Syria 10 (1929), p. 319-325 (Scripta varia, p. 814), et de Ch. PicARD, Mélanges syriens offerts à Monsieur René Dussaud, Paris (1939), p. 319-343, suivi par H. SeYrig, Syria 31, 1954, p. 85 (Antiquités syriennes 5, p. 104), et par Y. HaJJAR, Triade 1-2, p. 109-117. A. Farnoux, LIMC 6 (1992), p. 315, n 46, se contente de rappeler le mauvais état de conservation des reliefs. Photos récentes dans L. Nordiguian, Temples de l'époque romaine au Liban, Beyrouth (2005), p. 36-39.

16. Triade 1-2, n 130. Jupiter et Vénus apparaissent sur les autres faces de l'autel.

17. Triade $1-2, \mathrm{n}^{\circ} 108$.

18. J.-P. ReY-CoQuais, Topoi 9/2 (1999), p. 640, n 7, sur l'autel votif trouvé dans les ruines du grand temple de Qalaat Faqra, puis revu à 300 m à l'ouest du bâtiment : $\Delta \mathrm{lì}$

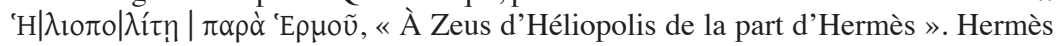
est bien ici le dieu et non un homme, étant donné la rareté relative de l'anthroponyme au Proche-Orient (cf. toutefois un exemple connu au Liban, à Nahlé, IGLS 6, 2891).

19. Baalbek 2, p. 22, fig. 38 (linteau); P. Collart et P. Coupel, L'autel monumental de Baalbek, Paris (1951), pl. 81, 3 ; 82, 13 ; 85, 34 (autel). Par imitation, le caducée ailé devient l'attribut de Zeus et d'Apis, les grands dieux d'Abila, sur l'autel de Brahlia conservé au Musée de Damas, cf. Y. HAJJAR, AAAS 27-28 (1977-1978), p. 187-195. 
Héliopolis quand firent naufrage les colonnes du temple de cette ville qui sont actuellement à Béryte ». Zeus demande à un dieu de porter ce message à Poséidon, le patron de Béryte : «Dis à Poséidon : "Il convient d'obéir à ses frères plus âgés ; tu te pares des colonnes de ma cour glorieuse et c'est inconvenant." Dis ces paroles en agitant trois fois la mer étincelante et il obéira. Mais s'il ne se soumet pas, qu'il craigne de me voir brûler la terre entière ; car rien, pas même la mer, n'éteint le foudre de Zeus ${ }^{20}$. » Il y a tout lieu de croire qu'il est ici question des colonnes de granite égyptien destinées à orner la cour du grand sanctuaire de Baalbek. P. Chuvin, qui l'admet, reconnaît que l'opposition des patrons de Béryte et d'Héliopolis recouvre celle des deux cités, mais il considère que Zeus passe par l'intermédiaire de Balmarcod, le dieu de Deir el-Qalaa ${ }^{21}$. À mon avis, un dieu héliopolitain convient mieux et Mercure paraît tout désigné en qualité de messager de Jupiter. Le jeune dieu de Baalbek se rapprocherait ainsi des anges du ProcheOrient, tels Mélicerte sur l'Hermon ou Malakbel, l'« Ange de Bel », à Palmyre ${ }^{22}$.

Le Mercure héliopolitain est donc un dieu original et distinct de Sol et de Bacchus. Il en va de même de ses alter ego libanais, qui lui ressemblent sans que l'on connaisse leur nom et qui n'appartiennent pas forcément à la triade

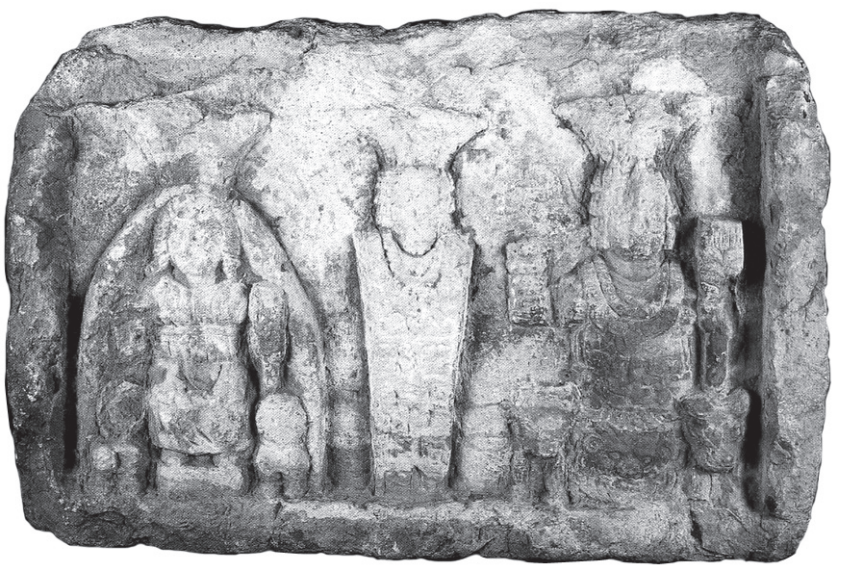

Fig. 5. - Bas relief de Fneidiq, Musée national de Beyrouth. Photo C. Doumet Serhal et al., Pierres et croyances, Beyrouth (1997), p. $124, n^{\circ} 77$.

20. Anthologie palatine 14, 75, éd. F. BufFì̀re, CUF, Paris (1970), p. 73 : Eỉı̇̀

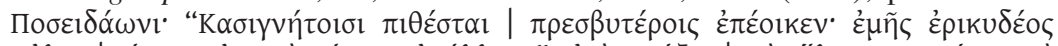

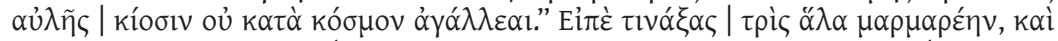

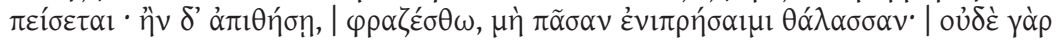

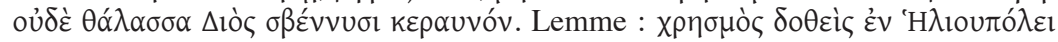

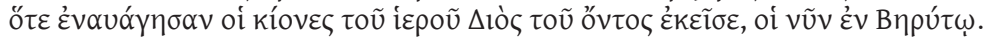

21. P. Chuvin, Mythologie et géographie dionysiaques, Clermont-Ferrand (1991), p. 218-221 (à qui j'emprunte la traduction de l'épigramme, tout en restituant sa valeur générale au premier vers), cf. M.-C. FAYANT, Nonnos de Panopolis, Les Dionysiaques. Chants XLI-XLIII, Paris (2006), p. 130-131.

22. J. Aliquot, BAAL 8 (2004), p. 304-305, à propos de Mélicerte. 
Fig. 6 - Bas-relief héliopolitain de Rome, Antiquarium du Palatin. Photo Triade 1-2, $\mathrm{n}^{\circ} 293$, pl. 114.

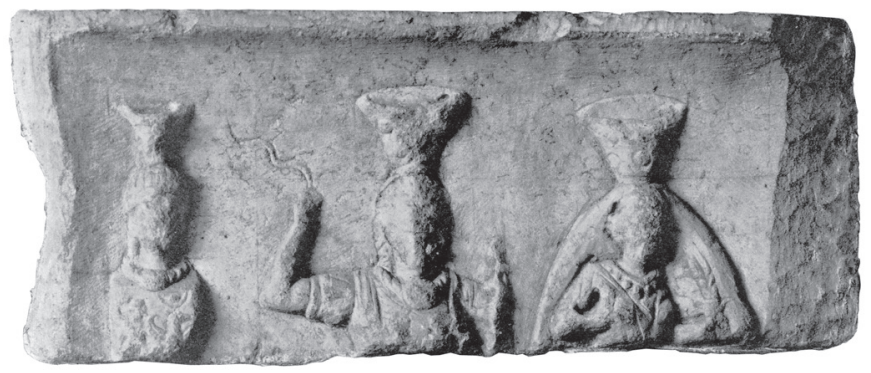

héliopolitaine, en particulier lorsqu'ils présentent des traits originaux ${ }^{23}$. Sur le bas-relief de Fneidiq (Liban-Nord), par exemple, la hiérarchie habituellement respectée dans l'iconographie héliopolitaine est renversée. On y trouve un groupe identique à celui de Baalbek, mais Mercure occupe le centre de la composition (Fig. 5), contrairement à ce que l'on voit sur les rares monuments qui réunissent les dieux héliopolitains, avec Jupiter au milieu, Vénus à gauche et Mercure à droite, conformément aux règles de préséance de la tradition romaine (Fig. O). Comme le note H. Seyrig, cet écart rappelle l'usage, notamment suivi dans le culte de Hiérapolis, de placer une enseigne sacrée portative entre Hadad et Atargatis (le onúïiov du De Dea Syria 33) ${ }^{24}$. Le sanctuaire éventuellement associé au monument de Fneidiq est situé dans la haute vallée du Nahr Abou Moussa. Entre la côte et la Békaa, la région appartient à l'arrière-pays d'Arca et d'Orthosie, qui puisent elles aussi dans le répertoire iconographique héliopolitain pour représenter leurs dieux. Les populations du littoral et de la montagne vénèrent ainsi des dieux formellement comparables à ceux de Baalbek.

Plus près de Baalbek, d'autres Mercures libanais différents de celui d'Héliopolis portent la marque du modèle héliopolitain. À Ham dans l'Antiliban,

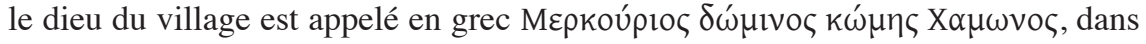

23. Y. HAJJAR, Triade 1-2, en convient à propos des nos 143 (Hermel), 144 (Jbab elHomr) et 342 (Niha). Des réserves similaires s'appliquent aux nos 130 (Béchouat), 135 (Douris), 136 (Fiké), 140141 (Harbata), ainsi qu'aux reliefs hermaïques de Yammouné et de Beit Jallouk, cf. C. Doumet-Serhal et al., Pierres et croyances, Beyrouth (1997), p. 86, $\mathrm{n}^{\circ} 45$, et L. Nordiguian, Temples de l'époque romaine au Liban, Beyrouth (2005), p. 224.

24. H. Seyrig, BMB 12 (1955), p. 25-28 (Scripta varia, p. 91-95). Sur le onuńiov hiérapolitain et les monuments apparentés, des enseignes sacrées de la glyptique syrienne aux vexilla de l'armée romaine, voir H. SEYRIG, Syria 37 (1960), p. 233246 (Antiquités syriennes 6, p. 79-92), H. Seyrig, Syria 49 (1972), p. 104-108, et J.L. Lightfoot, Lucian. On the Syrian Goddess, Oxford (2003), p. 446-449 et 540-547. L'utile mise au point de J.L. Lightfoot ne tient pas compte du fait que

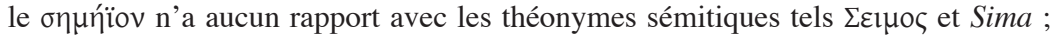
cf. J.T. MilıK, Biblica 48 (1967), p. 567-570, Dédicaces faites par des dieux, Paris (1972), p. 408-411, et P.-L. Gatier, Topoi 7/2 (1997), p. 761-762. 

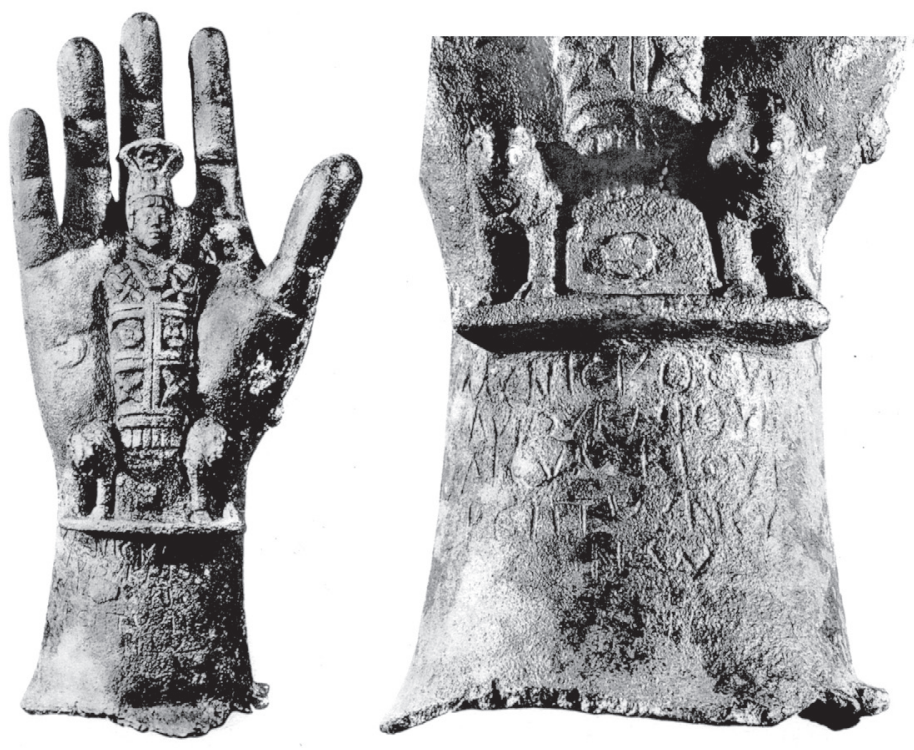

Fig. 7 - Main votive « de Niha », Musée du Louvre. Photo IGLS 6, 2930, pl. 47.

le texte qui commémore la construction de son temple en $172 / 3^{25}$; ce n'est sans doute pas un hasard si le nom d'homme Mrpkoúproc rencontre un petit succès près de Ham, dans la Békaa et en Abilène ${ }^{26}$. À Niha, les grands dieux Hadaranès et Atargatis, qui conservent des noms sémitiques, sont peut-être accompagnés d'un jeune parèdre semblable au Mercure héliopolitain. Placé dans la paume d'une main divine en bronze passant pour provenir de ce site, ce dernier prend la forme du terme, debout, de face, cuirassé sur un socle et flanqué de béliers : le visage imberbe, coiffé du calathos, paré d'un collier, il est enserré dans une gaine décorée de motifs stellaires et solaires, de croix et de rosaces; comme son homologue de Baalbek, il apparaît en position d'hypostase et de messager de la divinité suprême (Fig. 7) ${ }^{27}$. D'après les exemples cités, la romanisation des cultes passe soit par

25. C. Ghadban, Sociétés urbaines, sociétés rurales (éd. E. Frézouls), Strasbourg (1987), p. 232-233 (SEG 37, 1456).

26. IGLS 6, 2731 (Békaa) ; I. Syrie 1875 a (Souk Ouadi Barada) ; J.-P. ReY-CoquaIs, Egitto e storia antica dall'ellenismo all'età araba (éd. L. Criscuolo et G. GERACI), Bologne (1989), p. 612-613 (Nébi Abel).

27. H. SeYrig, Syria 31 (1954), p. 83, pl. 12 (Antiquités syriennes 5, p. 102), cf. IGLS 6, 2930, et N. Bel et P.-L. GATIER, Monuments Piot 87 (2008), p. 90-91, n 1, fig. 5-8. Rappelons que la provenance de la main « de Niha » n'est pas assurée. Dans le grand temple de Niha, la plateforme de l'adyton est ornée de l'ébauche d'un bas-relief où 
l'imitation de la nomenclature latine, soit par la formation d'une triade avec un dieu jeune semblable à Mercure.

\section{Sanctuaires et carrières de Mercure}

Le Mercure héliopolitain possède son propre sanctuaire hors-les-murs, au sud de Baalbek, sur la colline de Cheikh Abdallah. Tel qu'il est représenté sur les monnaies de la cité frappées sous Philippe l'Arabe, le temple qui s'élevait là est un périptère sur podium entouré d'un péribole, auquel mène un escalier monumental rupestre aménagé à flanc de colline ; dans le champ, la bourse et le caducée garantissent l'attribution du bâtiment à Mercure ${ }^{28}$. Après les travaux effectués sous la direction d'O. Puchstein, de D. Schlumberger et d'H. Kalayan, l'étude du site a repris en 2002 : sur le replat, des éléments du temenos et les fondations du temple ont été mis au jour ; K. Rheidt propose de restituer un temple hexastyle périptère d'ordre corinthien, ouvert au nord-est, dont le décor architectural rappelle notamment celui du petit temple du grand sanctuaire de Baalbek ${ }^{29}$. Le dossier épigraphique du lieu comprend sept textes où le nom du parèdre mineur de la triade héliopolitaine apparaît en latin et en grec, en toutes lettres ou en abrégé. Une dalle calcaire porte l'indication 'E $\rho \mu \mathrm{o}[\tilde{u}]$, « (propriété) d'Hermès ${ }^{30}$ ». Sur un rocher au sud de la colline, l'abréviation MER signale vraisemblablement que le dieu exploite un domaine adjacent au sanctuaire (cf. infra) ${ }^{31}$. À ces marques de propriété s'ajoutent des dédicaces émanant soit de colons romains issus de

J.-P. Rey-Coquais, Mélanges Pierre Levêque 6 (éd. É. Geny et M.-M. Mactoux), Besançon/Paris (1992), p. 252-258, reconnaît, à gauche d'un prêtre faisant une libation ou brûlant de l'encens sur un autel, la déesse Atargatis accompagnée d'un alter ego de Mercure chevauchant un bélier. Le thème serait conforme à l'imagerie grecque et romaine classique, cf. G. SIEBERT, LIMC 5 (1990), p. 310-311, n ${ }^{\text {os }} 258$ 259, et E. Simon, LIMC 6 (1992), p. 513, nos 139-144. Néanmoins, l'examen du relief sur le site me permet d'identifier plutôt Éros ailé sur un taureau, face à un dieu engainé qui pourrait être Hadaranès, dans une attitude semblable à celle du Jupiter héliopolitain. Voir aussi la photo de L. Nordiguian, Temples de l'époque romaine au Liban, Beyrouth (2005), p. 57.

28. Triade $1-2, \mathrm{n}^{\text {os }} 8486$.

29. Baalbek 1, p. 45-46 ; D. SCHLUMBERGER, BMB 3 (1939), p. 25-36 ; R. SAIDAH, BMB 20 (1967), p. 157-158 ; Triade 3, p. 347 ; K. RHeIDT, BAAL 9 (2005), p. 118-125 ; cf. les clichés de M. van Ess et T. Weber, Baalbek, Mayence (1999), p. 48, fig. 43a-b, et de L. Nordiguian, Temples de l'époque romaine au Liban, Beyrouth (2005), p. 43. Voir aussi Triade 1-2, n 90 : relief où subsistent la tête et la patte antérieure d'un bélier de profil en haut-relief.

30. IGLS 6, 2736 (Triade 1-2, $\mathrm{n}^{\circ} 88$ ).

31. Triade $1-2, \mathrm{n}^{\circ} 89$. 
familles en vue à Béryte et à Baalbek, celles des Afidenii ${ }^{32}$, des Statilii (ou Statii ${ }^{33}$ ) et des Tittii ${ }^{34}$, soit de fidèles au statut plus modeste (Fig. 8) ${ }^{35}$.

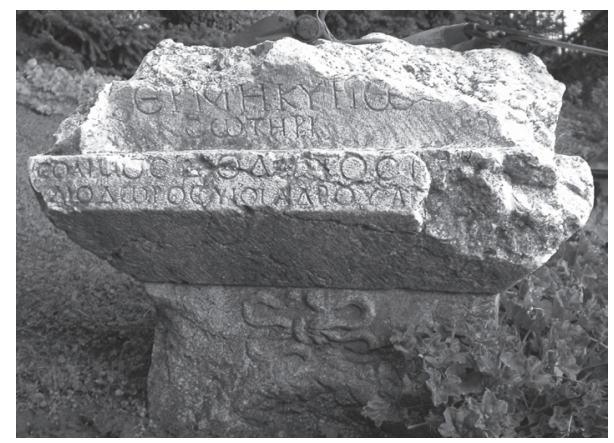

Fig. 8 - Dédicace à Hermès sur un autel du sanctuaire de Cheikh Abdallah à Baalbek. Collection privée à Beit Méry (Liban). Photo J. Aliquot (avril 2008).

32. IGLS 6, 2737 (Triade 1-2, $\mathrm{n}^{\circ}$ 83), sur un bloc de calcaire : Mercuri/o dom(ino),/ L(ucius) Afidenus / Stati [li]a[nus] / Val[ens].Le dédicant est identifiable ou apparenté à Lucius Afidénus, auteur de la dédicace de Baalbek à Mercure et à Apollon IGLS 6, 2738, et à Lucius Afidénus Valens, l'époux de Statia Rufilla, fille de Quintus, prêtresse du culte impérial honorée à Béryte, cf. H. SEYRIG, $B M B 1$ (1937), p. 84 (Scripta varia, p. 72).

33. H. Salamé-SARKis, Berytus 35 (1987), p. 131, n 8, sur le dé d'un autel : Mercur [i]/o dom(ino),/Stat(ilius) Afide/nus L(uci) f(ilius) Fab(ia tribu) Ru/fillus, praef(ectus) / fab [rum], tr(ibunus) mil(itum) / leg(ionis) V Mac(edonicae),/ v(otum) l(ibens) a(nimo) s(olvit), « au seigneur Mercure, Statilius Afidénus Rufillus, fils de Lucius, de la tribu Fabia, préfet des ouvriers, tribun des soldats de la Ve légion Macedonica, s'est acquitté de son vœu de bon gré ». L. 2. Le gentilice Statilius est attesté à Béryte, Deir el-Qalaa et Baalbek, cf. H. SeYrig, Syria 12 (1931), p. 320-321 (Antiquités syriennes 1, p. 56), et IGLS 6, 2793-2795 ; mais on peut aussi lire Stat(ius), nom connu à Béryte (n. 32) et à Baalbek $(S E G 40,1411)$; en revanche, il faut écarter l'idée d'H. Salamé-Sarkis, qui développe le cognomen Stat(ilianus) à la place du gentilice.

34. H. Salamé-Sarkis, Berytus 35 (1987), p. 130, n 7 (texte en majuscules), sur la corniche d'un autel : Mercu[ri]/o do[m(ino)] / Tittia / Severa / Rufilli / [- - ]. Autres Tittii au Liban : IGLS 6, 2723, cf. J.-P. REY-CoQuAIs, REA 82 (1980), p. 132 (dédicace de Caius Tittius Raaeus au Jupiter héliopolitain, à Baalbek) ; CIL 3, 6684 (dédicace de Marcus Tittius Re[...], à Deir el-Qalaa).

35. H. Salamé-Sarkis, Berytus 35 (1987), p. 130, nº 6 (SEG 38, 1562), sur la corniche d'un autel dont le dé est orné du caducée dans une couronne (revu en 2008 chez un

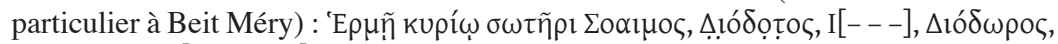
viò̀ $A \delta \rho o v, \grave{\alpha}[v \varepsilon ́ \theta \eta \kappa \alpha v]$, « à Hermès, seigneur sauveur, Soaimos, Diodotos, [...] et Diodoros, les fils d'Adros, ont dédié (l'autel). » L. 4. À la fin, je restitue le verbe

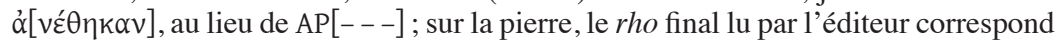
à une épaufrure de la pierre (Fig. 8). D'après IGLS 6, 2735 (Triade 1-2, n 87), la dédicace assez fruste $\Delta e o$ Mercyri $[o]$, découverte au pied de la colline, serait de provenance incertaine ; H. SEYRIG, BMB 1 (1937), p. 84 (Scripta varia, p. 72), n'hésite pas à la rapporter au sanctuaire périurbain de Mercure, sans doute avec raison. 


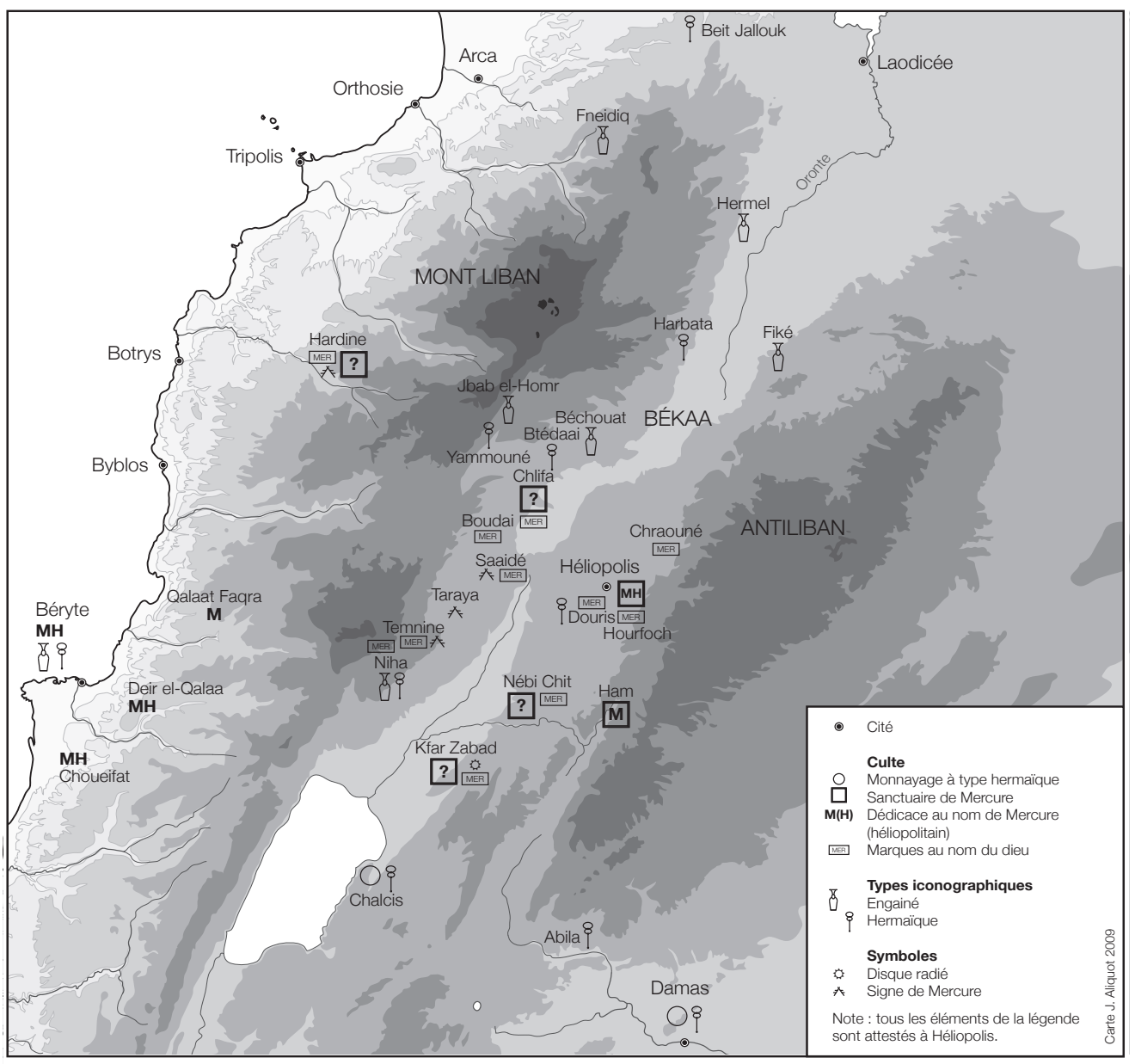

Fig. 9 - Mercure au Liban.

Il est difficile d'attribuer au Mercure héliopolitain d'autres sanctuaires que celui de Cheikh Abdallah, en dehors de ceux qu'il partage avec Jupiter et Vénus, à Baalbek, Béryte et peut-être Yammouné. Mercure est avant tout lié aux carrières du Liban, où $\mathrm{C}$. Ghadban a repéré des dizaines d'inscriptions, avec les lettres latines $M E R$ associées à trois symboles (étoile, disque radié, « signe de Mercure » en forme de $\mathrm{V}$ évasé barré de traits parallèles) et à des lettres grecques (sigma carré inversé, phi) ${ }^{36}$. Ces marques se concentrent au sud de la Békaa et dans les

36. C. Ghadban, Nouvelles inscriptions, Lyon (1978), p. 231-246, et Sociétés urbaines, sociétés rurales (éd. E. Frézouls), Strasbourg (1987), p. 236-237. Pour les marques de Hardine, cf. J. Yasmine, dans ce volume. 


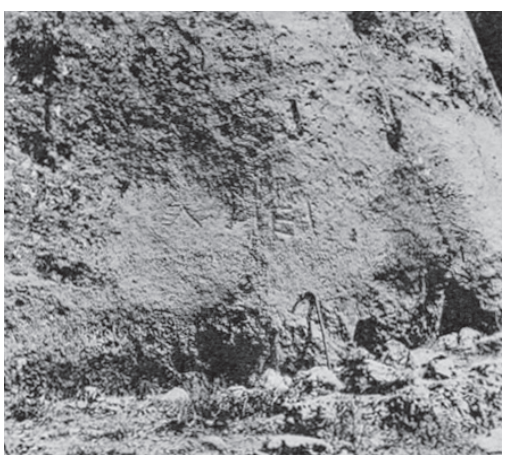

Fig. 10 - « Signe de Mercure » et marque $M E R$, dans la carrière proche du temple romain de Nébi Chit. Photo IGLS 6, 2972A, pl. 52.

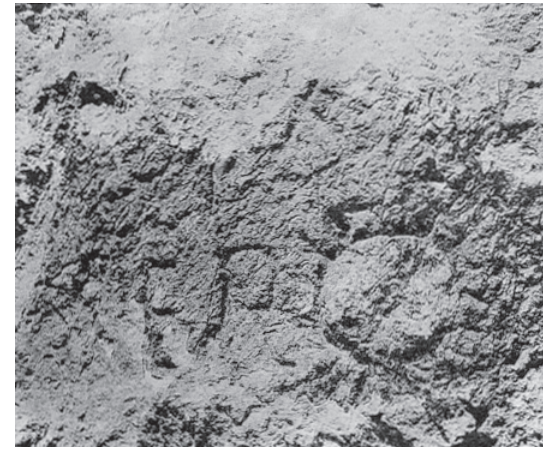

Fig. 11 - Marque MER et disque radié, sur le Jabal Terbol. Photo IGLS 6, 2977, pl. 52 .

montagnes voisines (Fig. 9-11). On les trouve d'abord dans les parages immédiats de Baalbek, au sud de la colline de Cheikh Abdallah, à Chraouné, à Douris et à Hourfoch. Plus au sud, dans l'Antiliban, des inscriptions semblables apparaissent à Nébi Chit, dans la carrière proche du sanctuaire romain, et sur le Jabal Terbol, à $2 \mathrm{~km}$ au nord des temples de Kfar Zabad. Sur le versant oriental du Mont Liban, du nord au sud, on en repère encore à Chlifa, avec une marque isolée au sud de la colline du temple romain, à Boudai, avec deux marques isolées, l'une à l'endroit, l'autre à l'envers, entre Mazraat el-Dellil et Saaidé, dans une carrière, avec trente-cinq marques associées au « symbole de Mercure » et à d'autres signes (non décrits), entre Taraya et Chmistar, dans une carrière, à Temnine el-Faouqa, dans les carrières à l'ouest du village, et entre Niha et Hosn Niha, sur le front de taille d'une carrière. Les exemplaires de Hardine, dans la carrière en contrebas du temple romain, sont les seuls repérés à ce jour à l'ouest des crêtes du Mont Liban. De manière remarquable, enfin, on retrouve les lettres $M E R$ à Baalbek, sur le site de la Qalaa, dans la cour du grand sanctuaire, sur des colonnes ou fragments de colonnes en granite rose d'Assouan, et, entre les deux temples, sur le lit d'attente d'une base de colonne inachevée, puis dans la zone archéologique de Bostan elKhan, sur la base d'une colonne. Aucune marque semblable ne paraît attestée ni en Égypte, ni ailleurs dans l'Empire.

Avant les trouvailles de C. Ghadban, les seules marques connues étaient celles de la colline de Cheikh Abdallah, de Chlifa, du Jabal Terbol et de Nébi Chit. Comme elles se trouvaient à proximité de temples romains, elles étaient censées délimiter le domaine des sanctuaires de Mercure ${ }^{37}$. Leur association aux

37. R. Mouterde, MUSJ 29 (1951-1952), p. 60-63, et MUSJ 36 (1959), p. 73-74, nº 13 ; IGLS 6, 2912, 2972, 2977 ; Triade 1-2, ${ }^{\circ \text { s }} 89,137,150$. Cf. également IGLS 6, 2824A, sur le lit d'attente d'une base de colonne inachevée, entre le grand temple et le petit temple de la Qalaa. 
sanctuaires du Liban semble aujourd'hui peu probante, compte tenu de la grande diversité de leurs supports. Rien ne permet par ailleurs de suivre C. Ghadban lorsqu'il voit, dans toutes ces inscriptions, la trace de « Mercuriens » appartenant à une « entreprise placée sous le patronage du Mercure Héliopolitain et exploitant les carrières avec des moyens énormes $»{ }^{38}$. Pour autant, les marques semblent bien se rapporter à une entreprise de construction qui serait liée à Mercure et qui œuvrerait sur le territoire de Béryte et d'Héliopolis ${ }^{39}$. Elles ne sont pas des dédicaces semblables à celles, explicites, que l'on connaît dans les carrières de l'Empire ${ }^{40}$; elles ne servent pas à l'assemblage de blocs, puisqu'on les trouve à la fois sur des blocs isolés et sur des fronts de taille. À mon avis, ces marques indiquent le nom du propriétaire des pierres où elles sont gravées, avec deux usages distincts : sur des matériaux d'importation, tel le granite syénite, leur auteur les applique pour éviter de confondre ses blocs avec ceux acquis par d'autres acheteurs et débarqués en un seul et même endroit, sans doute à Béryte ; dans les carrières libanaises, il distingue des zones dont l'exploitation lui est concédée. Si l'on cherche à identifier le bénéficiaire de telles opérations, on peut penser au prince, mais uniquement pour les pierres d'importation car, à la différence du granite égyptien, le calcaire de la Békaa, n'ayant rien de précieux, ne saurait faire partie du patrimonium Caesaris : ce sont vraisemblablement les cités de Béryte et d'Héliopolis qui tirent profit des besoins en pierre locale engendrés par les chantiers de Baalbek. Quant au commanditaire des blocs aux lettres $M E R$, il s'agit probablement du Mercure héliopolitain, car la fréquence de l'abréviation $M E R$ dans les dédicaces latines garantit la lecture du théonyme Mer(curius), ici déclinable au génitif, Mer(curi). Le Mercure héliopolitain patronnerait ainsi la construction religieuse à Baalbek, en exploitant les carrières civiques et en important des pierres d'Égypte. On comprend que, dans l'oracle de l'Anthologie, Jupiter ait choisi de faire appel au jeune dieu pour récupérer ses colonnes.

Le probable patronage du Mercure héliopolitain sur la construction religieuse invite à la prudence : il est possible que le dieu possède les sanctuaires de Hardine, Chlifa, Nébi Chit et Kfar Zabad, près desquels on trouve les marques à son nom, mais non ceux de Niha, où les dédicaces s'adressent à d'autres divinités. Hors de Baalbek, en définitive, le sanctuaire libanais attribuable de façon certaine à Mercure est celui de Ham, dans une formule analogue, mais différente du culte héliopolitain.

38. C. Ghadban, Sociétés urbaines, sociétés rurales (éd. E. Frézouls), Strasbourg (1987), p. 236-237.

39. Je n'en ai repéré aucune dans les carrières inédites au sud de Kamid el-Loz, sur le territoire antique de Sidon, au cours de la prospection épigraphique menée en vue de la publication du tome 11 des $I G L S$ consacré au Mont Hermon (Beyrouth [2008]).

40. Ainsi à Enesh sur l'Euphrate : F. Cumont, Études syriennes, Paris (1917), p. 159166. 


\section{Mercure sans Sol ni Bacchus}

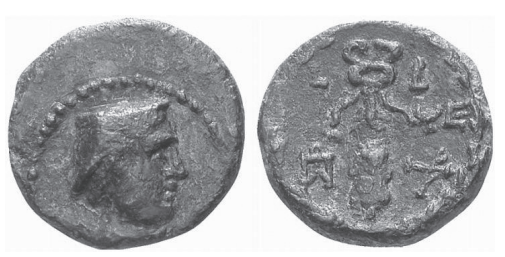

Fig. 12 - Monnaie de bronze de Ptolémaios de Chalcis : au droit, tête d'Hermès casqué ; au revers, caducée, monogrammes du dynaste et date ('̌́ ${ }^{\prime}$ ous $\beta^{\prime}=63 / 62$ av. J.C.). Collection J.-P. Righetti, exemplaire publié le 10 octobre 2006 sur le site CoinArchives. com, semblable à D. HERMAN, Israel Numismatic Research 1 (2006), p. 62, $\mathrm{n}^{\circ} 6, \mathrm{pl} .7$. dieu pâtre assimilé à Hermès que se forment les légendes qui, dès l'époque hellénistique, font de la Békaa et de l'Antiliban une petite Arcadie aux marges de Béryte, Damas et Césarée-Panéas ${ }^{43}$. Sous Auguste, des Romains sont lotis dans la Békaa. S'ils adoptent le culte local en identifiant Hermès à Mercure, ils peuvent invoquer Mercure pour des raisons qui leur sont propres : les Romains choisissent volontiers Mercure comme l'équivalent de dieux qui leur paraissent étranges ${ }^{44}$.

41. Pour les monnaies frappées au nom de Ptolémaios en 73/2 et en 63/2 av. J.-C., avec la tête d'Hermès casqué à droite, à l'avers, et le caducée, au revers : A. KindLER, in T. Hackens et G. Moucharte (éds), Actes du XI Congrès International de Numismatique 1, Louvain (1993), p. 286-287, nº 4 et 6a ; D. Herman, INJ 14 (20002002), p. 85-86, $\mathrm{n}^{\text {os }} 3$ et 6 , pl. 13, 16-17 ; D. Herman, Israel Numismatic Research 1 (2006), p. 60-62, nos 3 et 6, pl. 67. Sur les relations entre les Mennaïdes et Baalbek, cf. J. AliQuot, MUSJ 56 (1999-2003), p. 218-219.

42. $R P C 1, \mathrm{n}^{\text {os }} 4784(33 / 2$ av. J.-C.) et 4788 (30/29 av. J.-C.) : revers au type d'Hermès tenant le caducée.

43. Je développe ce thème dans ma thèse sur La vie religieuse au Liban sous l'empire romain, BAH 189, Beyrouth (2009).

44. C'est la raison pour laquelle César, Guerre des Gaules 6, 17, 1, appelle Mercurius le dieu principal des Gaulois. Tacite, Germanie 9, 1, nomme le Germanique Wodan de la même manière. Sur le culte de Mercure en Gaule et en Germanie, G. BauchHENSS, LIMC 6 (1992), p. 547-554, et W. VAN ANDRINGA, La religion en Gaule romaine, Paris (2002), p. 135-137. 
Ils jouent en outre un rôle décisif dans la formation de la triade héliopolitaine, ce groupe divin aussi strictement hiérarchisé que la triade capitoline.

Mercure n'étant ni Sol ni Bacchus, il convient de préciser la place de ces deux derniers dieux au Liban. Selon H. Seyrig, qui rejette l'affirmation problématique d'un Macrobe succombant trop facilement au panthéisme solaire, le Jupiter héliopolitain n'a jamais été assimilé au Soleil ${ }^{45}$. Or, ce dieu est au contraire la seule divinité de la triade à avoir la tête radiée, sur une série de gemmes et d'intailles (Fig. 13) ${ }^{46}$. Restons prudents : l'interprétation du culte ne peut dépendre de ces modestes manifestations de la piété privée. Néanmoins, on accordera à Macrobe que la posture du dieu, qui fait toute son originalité et qui est

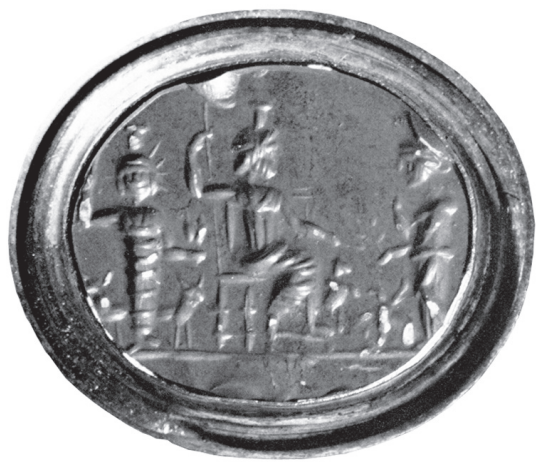

Fig. 13 - Intaille de jaspe rouge, British Museum : Jupiter héliopolitain engaîné et radié, à gauche de Sérapis trônant et de Némésis debout. Photo Triade 1-2, $\mathrm{n}^{\circ} 312$, pl. 123. un trait constant de l'imagerie officielle, rappelle en tout point celle du cocher solaire brandissant le fouet. Ajoutons que cette solarisation peut correspondre à un héritage hellénistique : l'octroi du nom d'Héliopolis à Baalbek, déjà attesté chez Strabon, implique la pratique d'un culte héliaque dans le sanctuaire de la Békaa, dès l'époque hellénistique. Au Liban, cette solarisation s'intégrerait par ailleurs dans un ensemble de témoignages attestant la pratique des cultes héliaques, dont $\mathrm{H}$. Seyrig a montré le caractère traditionnel en milieu arabe ${ }^{47}$. Il est vrai que l'on ne trouve aucun trait héliaque sur les monnaies mennaïdes, mais l'hellénisation du dieu peut masquer les caractéristiques qui apparaissent dans l'imagerie héliopolitaine. En somme, le prototype du Jupiter héliopolitain serait un grand dieu vénéré sous un aspect solaire par les Arabes du Liban à l'époque hellénistique, assimilé à Zeus par les dynastes de Chalcis au premier siècle avant notre ère et placé par les Romains sous le nom de Jupiter aux côtés de Vénus et de Mercure au début de l'Empire.

En ce qui concerne Bacchus, il semble probable que ce dieu soit accueilli à Baalbek comme à Béryte. Plusieurs témoignages illustrent la floraison de son culte à Béryte. Dans l'hymne où il énumère les divinités poliades (Hermès, Aphrodite et son cortège, Artémis, les Néréides, Zeus, Arès, les Grâces, la nymphe Béroé), Nonnos de Panopolis désigne la ville comme le « délicieux séjour

45. H. SeYrig, Syria 48 (1971), p. 345-348, cf. Macrobe, Saturnales 1, 23, 10-12.

46. Triade $1-2, \mathrm{n}^{\text {os }} 156,176,312,319,324$.

47. H. SeYrig, Syria 48 (1971), p. 337-373. 


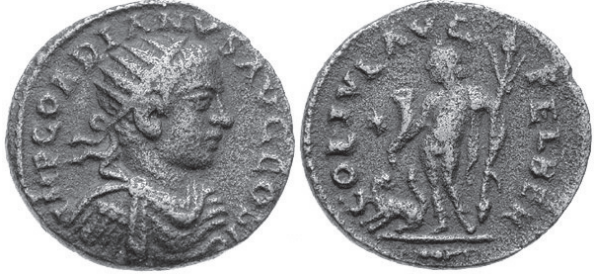

Fig. 14 - Monnaie de bronze de Béryte (239241) : au droit, buste de Gordien III à droite ; au revers, Bacchus debout, imberbe, appuyé sur le thyrse et abreuvant sa panthère de vin. Collection privée, exemplaire publié sur le site WildWinds.com, semblable à G.F. HILL, A Catalogue of Coins in the British Museum. Phoenicia, Londres (1910), p. 89, n $^{\circ} 245$.

de Bacchos »; et chez cet auteur qui consacre trois chants de ses Dionysiaques aux traditions locales, Bacchus est le second grand dieu de la cité ${ }^{48}$. D'autres monuments invitent à partager l'enthousiasme du poète : Bacchus figure au revers des monnaies de Béryte, peut-être déjà sous Hadrien, sûrement sous son aspect juvénile sous Gordien III (Fig. 14) ${ }^{49}$; une inscription lacunaire commémore l'érection de sa statue en ville, en le nommant Liber Pater en latin ${ }^{50}$; Leucothéa, la nourrice du dieu, est elle aussi invoquée en latin à Béryte, à la suite de la triade héliopolitaine ${ }^{51}$; enfin, l'onomastique coloniale témoigne de l'importance locale du culte bachique, en particulier dans la gens Statilia, dont deux représentants portent le cognomen Bromiacus ou Brumiacus, dérivé de Bromius, surnom de Bacchus ${ }^{52}$. Par conséquent, rien n'empêche que Bacchus, vénéré à Béryte, possède

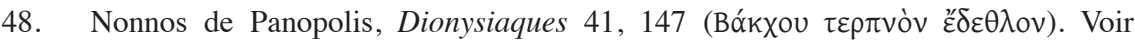
P. Chuvin, Mythologie et géographie dionysiaques, Clermont-Ferrand (1991), p. 209-210.

49. C. Augé et P. Linant de Bellefonds, LIMC 3 (1986), p. 519, nº 44 (Hadrien) : exceptionnellement barbu, Bacchus, de profil à droite, couronné de feuillage et vêtu d'une tunique, s'appuie sur le thyrse de la main gauche et tient le canthare dans la main droite ; à ses pieds repose la panthère. G.F. HiLl, A Catalogue of Coins in the British Museum. Phoenicia, Londres (1910), p. 88-89, $\mathrm{n}^{\text {os }}$ 241-244 (Gordien III) : debout, de face, nu et déhanché, le dieu lève le bras droit au-dessus de sa tête couronnée de feuillage et tournée à droite; de sa main gauche, il tient un petit personnage, peut-être un Satyre ; à gauche, la panthère repose, la tête tournée ; à droite, une vigne s'enroule autour d'une hampe ; dans le champ, une grappe de raisin. Ibid., p. 89, n $^{\text {os }}$ 245-249 (Gordien III) : Bacchus debout, imberbe, s'appuie sur le thyrse et abreuve sa panthère de vin.

50. R. CAGnat, Syria 5 (1924), p. 111, n 7. Toujours à Béryte, la dédicace d'un autel, CIL 3, 163, semble s'adresser à Liber : Lucia Polla, L(ibero) A(ugusto) s(acrum),/ v(otum) l(ibens) a(nimo) s(olvit), «Lucia Polla, (en offrant ce) monument consacré à Liber Auguste, s'est acquittée de son vœu de bon cœur. »

51. Dédicace inédite conservée au Musée national de Beyrouth.

52. H. SEYRIG, Syria 12 (1931), p. 320-321 (Antiquités syriennes 1, p. 56), à Béryte et à Deir el-Qalaa. Dès l'époque hellénistique, les noms théophores bachiques sont assez courants chez les Phéniciens, notamment ceux de Béryte et de Tyr, comme l'observe H. SEYRIG, Syria 31 (1954), p. 71-72 (Antiquités syriennes 5, p. 90-91). 
le petit temple de Baalbek, selon l'idée d'O. Puchstein émise dès 1902 à la suite du dégagement de la Qalaa puis abandonnée à grand renfort d'exégèses alambiquées ${ }^{53}$.

Le succès local du culte bachique n'aurait rien de spécifiquement libanais. Dionysos est nommément honoré au Proche-Orient sous l'Empire, à Byblos, Scythopolis et Sidon ${ }^{54}$. Contrairement à ce qu'on a pris l'habitude d'affirmer à la suite d'H. Seyrig, les monnaies dionysiaques du Proche-Orient romain peuvent être considérées comme des monuments attestant la pratique du culte bachique dans de nombreuses cités (Antioche, Apamée, Béryte, Botrys, Canatha, Capitolias, Césarée, Damas, Esbous, Jérusalem, Laodicée-sur-mer, Raphia, Scythopolis, Sidon, Tyr ${ }^{55}$. Dans ce contexte, il est peu probable que Bacchus prête son apparence à un dieu phénicien à Béryte. À l'époque hellénistique, Dionysos conserve en Phénicie l'apparence d'un dieu barbu d'âge mûr, ce qui indique que les Phéniciens sont moins séduits par les thèmes de sa jeunesse et de son triomphe que par les aspects chtoniens de son culte (conformes à la thématique funèbre des mythes et des cultes de leurs dieux souffrants, toujours prégnante à Sidon en 59/8 av. J.-C., d'après la dédicace à Dionysos cadméen du sanctuaire d'Echmoun) ${ }^{56}$. Dans la colonie de Béryte, le succès de Bacchus rappelle plutôt le regain de popularité que connaissent les mystères dionysiaques depuis que César les a officiellement réintroduits à

53. O. Puchstein et al., JDAI 17 (1902), p. 99, et Baalbek 2, p. 85, exclusivement d'après le décor du temple. Autres hypothèses : H. ThiERsch, Nachrichten von den königlichen Gesellschaft der Wissenschaften zu Göttingen, philologisch-historische Klasse (1925), p. 3-8 (Vénus, alias Atargatis) ; H. SeYrig, Syria 10 (1929), p. 318 (Scripta varia, p. 7), suivi par R. Dussaud, Syria 23 (1942-1943), p. 58 (la triade) ; Triade 3, p. 339-341 (Mercure-Bacchus) ; K.S. FreYBerger, DaM 12 (2000), p. 118119 (Jupiter assimilé au Soleil).

54. H. SEYrig, Syria 31 (1954), p. 68-73 (Antiquités syriennes 5, p. 86-91) (Byblos) ; L. Di Segni, Scripta Classica Israelica 16 (1997), p. 139-161 (Scythopolis); R. Wachter, Das Eschmun-Heiligtum von Sidon (éd. R.A. Stucky), Bâle (2005), $\mathrm{n}^{\circ}$ 5, cf. P.-L. GATIER, Bull. épigr. 2006, 461 (Bostan ech-Cheikh).

55. Inventaire provisoire établi d'après C. Augé et P. Linant De Bellefonds, LIMC 3 (1986), p. 514-531 ; ajouter $R P C 1, \mathrm{n}^{\text {os }} 4347-4354,4370$ (Apamée, époque augustéenne) et 4806 (Damas, 65/6 apr. J.-C.).

56. C. Augé et P. Linant de Bellefonds, LIMC 3 (1986), p. 518-519, n ${ }^{\text {os }} 4243$, pour les monnaies de Sidon au Dionysos barbu (II ${ }^{\mathrm{e}}$ s. av. J.-C.). Parmi les sculptures de Bostan ech-Cheikh représentant Dionysos, R.A. Stucky, Die Skulpturen aus dem Eschmun-Heiligtum bei Sidon, Bâle (1993), p. 28, 59-60, distingue les productions locales hellénistiques, où le dieu est barbu ( ${ }^{\text {os }}$ 81-84), des œuvres d'époque romaine, où il prend un aspect juvénile (n $\left.{ }^{\text {os }} 85-88\right)$. L'identification de l'Echmoun sidonien à Dionysos reste conjecturale. Elle pourrait procéder de spéculations sur les souffrances du dieu phénicien. 
Rome ${ }^{57}$. Les Romains lotis dans la Békaa partagent alors l'engouement des nouveaux fidèles du dieu de la vigne au Proche-Orient.

\section{Conclusion}

Mercure doit être dévêtu de son habit solaire et mystique pour être rendu à ses fidèles, les bergers de la montagne libanaise et les colons romains de Béryte. Son originalité illustre la contamination réciproque de différentes traditions religieuses sous l'Empire romain. À l'époque où ils s'installent au Liban, les Romains ne se contentent pas d'interpréter son culte : s'ils s'en accommodent, s'ils l'adoptent même pour des raisons qui leur sont propres, tout comme les Perses et les Grecs l'ont fait avant eux, ils sont aussi à l'origine de la réorganisation du panthéon héliopolitain, cet événement préludant à la propagation des cultes de Baalbek dans l'ensemble du monde romain.

\section{Appendice : Hermès au Proche-Orient}

Hermès prête parfois son nom à d'autres dieux syriens que le Mercure héliopolitain et ses alter ego libanais. Ainsi, selon le philosophe Jamblique, dont l'empereur Julien rapporte les dires dans son discours prononcé à Antioche en 362 apr. J.-C., les habitants d'Édesse l'identifieraient à Monimos, l'un des deux parèdres mineurs d'Hélios ${ }^{58}$. La documentation archéologique et épigraphique ne laisse toutefois jamais supposer que les fidèles syriens partagent de telles interprétations ${ }^{59}$. Pour ces derniers, Hermès semble rester un dieu grec.

Dès l'époque hellénistique, le culte d'Hermès fleurit dans les cités grecques de la Tétrapole syrienne : en témoignent les poids d'Antioche, de Séleucie et de Laodicée-sur-mer, qui portent l'image du caducée ou du dieu-terme en qualité de patron de l'agora ${ }^{60}$. C'est encore dans la même région, à Antioche, que l'on trouve sous l'Empire romain le seul sanctuaire de l'Hermès grec

57. Selon la glose de Servius sur Virgile, Bucoliques 5, 29 : Caesarem [...] quem constat primum sacra Liberi Patris transtulisse Romam, « il est établi que César fut le premier à acclimater à Rome les mystères de Liber Pater ». Cf. R. TuRCAN, Hommage à la mémoire de Jérôme Carcopino, Paris (1977), p. 317-325.

58. Julien, Discours 11, 34 .

59. Contra F. ZAYADINE, LIMC Suppl. (1997), p. 616-619, qui, suivant d'autres auteurs, rapporte divers témoignages hermaïques de Jordanie, de Mésopotamie et de Palmyrène aux divinités al-Kutbay et Ruday. Cf. également H.J.W. DriJvers, Cults and beliefs at Edessa, Leyde (1980), p. 146-174.

60. H. SeYrig, $B M B 8$ (1949), p. 42, n 8 (Antioche), 46, n 7 (Séleucie) (Scripta varia, p. 372 et 376) ; P.-L. GATIER, Syria 63 (1986), p. 375-378 (Laodicée, Antioche). 
attesté à ce jour au Proche-Orient ${ }^{61}$. Ailleurs, le dieu est aussi le patron du gymnase. En Phénicie, à Arados, il est associé à Héraclès-Melqart dans la dédicace bilingue grecque et phénicienne d'un gymnasiarque, en 25/4 av. J.-C. ; contrairement au nom d'Héraclès, qui est traduit par mlqrt, son nom est transcrit phonétiquement, probablement parce que les Aradiens ne lui connaissent aucun équivalent en phénicien ${ }^{62}$. On rapprochera ce texte des monnaies hermaïques frappées au II $^{\text {e }}$ s. av. J.-C. par Marathos, dans la pérée aradienne ${ }^{63}$. Hermès et Héraclès sont également honorés ensemble à Tyr par un éphèbe en 188/7 av. J.C., mais cette fois aux côtés des souverains séleucides Antiochos III et Séleucos IV ${ }^{64}$. Sous l'Empire

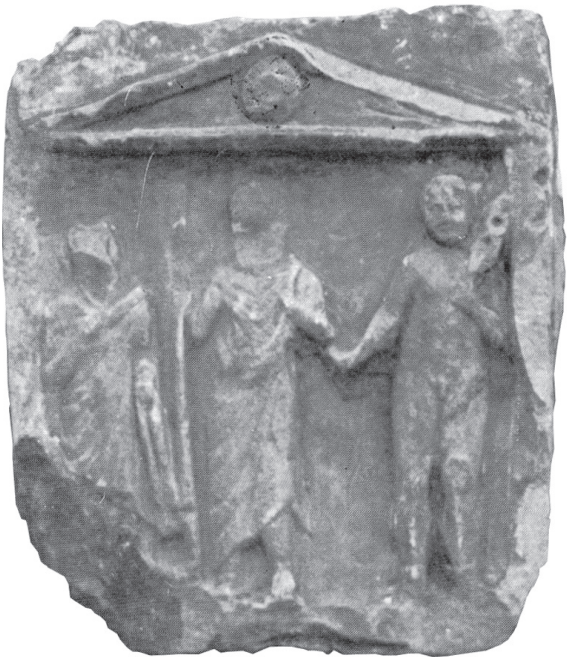

Fig. 15 - Monument funéraire de Baalbek, avec Hermès psychopompe. Photo Triade 1-2, n 6, pl. 3 . romain, Hermès reparaît encore dans le contexte évocateur de l'univers du gymnase et de la palestre. L'épigramme funéraire pour le jeune Philinos à Apamée l'associe aux Muses ${ }^{65}$. À Palmyre, les deux bas-reliefs remployés dans le mur médiéval fermant les propylées du sanctuaire de Bel portent son image et celle d'Héraclès ; ils ont pu orner un gymnase ou une palestre ${ }^{66}$.

Dieu du passage, Hermès escorte enfin les Syriens sur le chemin des enfers. Les monuments qui le présentent dans un contexte funèbre sont nombreux

61. Libanios, Discours 15, 79 ; Malalas, Chronographie 13, 3, cf. G. Downey, A History of Antioch in Syria, Princeton (1961), p. 349-350. Monnaies d'Antioche avec le caducée au I $\mathrm{I}^{\mathrm{er}}$ s. apr. J.-C., puis avec l'image du dieu au III ${ }^{\mathrm{e}} \mathrm{s}$. apr. J.-C. Voir F.W. NorRIS, ANRW 2, 18.4 (1990), p. 2350-2351 et 2358, avec les références.

62. IGLS 7, 4001 .

63. F. Duyrat, Les monnayages syriens (éd. C. Augé et F. Duyrat), Beyrouth (2002), p. 30-32.

64. J.-P. Rey-CoQuaIs, Inscriptions grecques et latines de Tyr, Beyrouth (2006), p. 17$18, \mathrm{n}^{\circ} 1$.

65. IGLS 4, 1350 .

66. H. SEYRIG, Syria 24 (1945), p. 75-76, n 17 (Antiquités syriennes 4, p. 14-15). 
au Proche-Orient ${ }^{67}$. Les évoquer tous serait aussi fastidieux qu'inutile. Je me contenterai d'attirer l'attention sur un témoignage méconnu. À Baalbek, une stèle funéraire semble classée par erreur dans les monuments relatifs à la triade héliopolitaine (Fig. 15) : on y découvre, sous un fronton, à l'intérieur d'un édicule, un bas-relief représentant un homme, le défunt, entre une pleureuse voilée et un Hermès psychopompe ${ }^{68}$.

\section{Julien Aliquot \\ Institut français du Proche-Orient \\ UMIFRE 6 (CNRS/MAE)}

\section{Bibliographie}

\section{Abréviations}

AE : L'Année épigraphique, RA (1888-1961), puis sous la forme de vol. indépendants, Paris.

ANRW : Aufstieg und Niedergang der römischen Welt (éd. W. HaAse et H. TemporinI), Berlin/New York (depuis 1972).

Antiquités syriennes : H. SeYrIG, Antiquités syriennes, Paris (1934-1966).

$B A A L$ : Bulletin d'archéologie et d'architecture libanaises, Beyrouth.

Baalbek: T. WiEgAND (éd.), Baalbek, Berlin/Leipzig (1921-1925).

BMB : Bulletin du Musée de Beyrouth, Beyrouth.

Bull. épigr. : Bulletin épigraphique de la RÉG (depuis 1888).

CIL : Corpus inscriptionum latinarum, Berlin (depuis 1863).

I. Syrie: W.-H. Waddington, Inscriptions grecques et latines de la Syrie, Paris (1870).

IGLS : Inscriptions grecques et latines de la Syrie, Paris, puis Beyrouth (depuis 1929).

LIMC : Lexicon iconographicum mythologiae classicae, Zürich/Munich, puis Düsseldorf (1981-1999).

MUSJ : Mélanges de l'Université Saint-Joseph, Beyrouth.

RPC : Roman Provincial Coinage, Londres/Paris (depuis 1992).

Scripta varia: H. SEYRIG, Scripta varia, Paris (1985).

SEG : Supplementum epigraphicum graecum, Leyde, Alphen aan den Rijn, puis Amsterdam.

67. Antioche : H. SEYRIG, Syria 20 (1939), p. 314, n 16, fig. 8 (Antiquités syriennes 3 , p. 45). Massyaf : F. Chapoutier, Syria 31 (1954), p. 172-211 (IGLS 4, 1388). Tyr : M. Dunand, BMB 18 (1965), p. 551. Hauran : D. Sourdel, Les cultes du Hauran à l'époque romaine, Paris (1952), p. 109. Décapole : A. LichtenBerger, Kulte und Kultur der Dekapolis, Wiesbaden (2003), p. 79, 108, 125-126, 233, 268, 307, 322.

68. Triade $1-2, \mathrm{n}^{\circ} 6$, où Y. Hajjar identifie Jupiter entre ses deux parèdres. 
Triade 1-2 : Y. HAJJAR, La triade d'Héliopolis-Baalbek, Leyde (1977).

Triade 3 : Y. HAJJAR, La triade d'Héliopolis-Baalbek, Montréal (1985).

\section{Études}

Aliquot J., « Les Ituréens et la présence arabe au Liban du II siècle $a . C$. au Iv $\mathrm{e}^{\mathrm{e}}$ siècle $p . C$.», MUSJ 56 (1999-2003), p. 161-290.

Aliquot J., «Inscriptions grecques et antiquités de Haloua », BAAL 8 (2004), p. 301-314.

Aliquot J., La vie religieuse au Liban sous l'empire romain, BAH 189, Beyrouth (2009).

Augé C. et P. LinAnt de Bellefonds, « Dionysos (in peripheria orientali) », LIMC 3 (1986), p. 514-531.

BADRE L., « Les figurines de plomb de 'Ain al-Djoudj », Syria 76 (1999), p. 181-196.

Bauchienss G. et E. Simon, « Mercurius », LIMC 6 (1992), p. 500-554.

Bel N. et P.-L. Gatier, « Mains votives de la Phénicie romaine », Monuments Piot 87 (2008), p. 69-104.

Buffière F. (éd.), Anthologie grecque. Première partie. Anthologie palatine (Livres XIIIXIV), Paris (1970).

Cagnat R., «Inscriptions latines de Syrie », Syria 5 (1924), p. 108-112.

CHApouthier F., « Les peintures murales d'un hypogée funéraire près de Massyaf », Syria 31 (1954), p. 172-211.

Chuvin P., Mythologie et géographie dionysiaques, Clermont-Ferrand (1991).

Chuvin P. et M.-C. Fayant (éd.), Nonnos de Panopolis, Les Dionysiaques. Chants XLIXLIII, Paris (2006).

Collart P. et P. Coupel, L'autel monumental de Baalbek, Paris (1951).

Cumont F., Études syriennes, Paris (1917).

Di SEgNi L., «A Dated Inscription from Beth Shean and the Cult of Dionysos Ktistes in Roman Scythopolis », Scripta Classica Israelica 16 (1997), p. 139-161.

Doumet-Serhal C. et al., Pierres et croyances. 100 objets sculptés des Antiquités du Liban, Beyrouth (1997).

Downey G., A History of Antioch in Syria, Princeton (1961).

DriJvers H.J.W., Cults and beliefs at Edessa, Leyde (1980).

Dunand M., « Tombe peinte dans la campagne de Tyr », BMB 18 (1965), p. 5-51.

Dussaud R., « Temples et cultes de la Triade héliopolitaine à Ba'albek », Syria 23 (19421943), p. 33-77.

Duyrat F., «Les ateliers monétaires de la Phénicie du Nord à l'époque hellénistique », Les monnayages syriens (éd. C. Augé et F. DuYrat), Beyrouth (2002), p. 21-69.

FARnoux A., « Lykourgos I », LIMC 6 (1992), p. 309-319.

Freyberger K.S., «Im Licht des Sonnengottes. Deutung und Funktion des sogenannten "BacchusTempels" im Heiligtum des Jupiter Heliopolitanus in Baalbek », DaM 12 (2000), p. 95-133.

Ganschow T., « Heliopolis », LIMC 8 (1997), p. 614.

Gatier P.-L., « Deux poids syriens de la Fondation Piéridès », Syria 63 (1986), p. 375-378. 
GATIER P.-L., « Villages et sanctuaires en Antiochène autour de Qalaat Kalota », Topoi 7/2 (1997), p. 751-775.

Ghadban C., Nouvelles inscriptions et topographie de la Béqa' (Thèse de $3^{\text {e }}$ cycle, Université de Lyon 2/Institut Fernand Courby), Lyon (1978).

GhadBan C., « Observations sur le statut des terres et l'organisation des villages dans la Béqa“ hellénistique et romaine », Sociétés urbaines, sociétés rurales dans l'Asie Mineure et la Syrie hellénistiques et romaines (éd. E. Frézouls), Strasbourg (1987), p. 217-238.

HajJar Y., La triade d'Héliopolis-Baalbek, Leyde (1977).

HaJjar Y., « Une dédicace de Brahlia à Zeus et Apis », AAAS 27-28 (1977-1978), p. 187195.

HajJar Y., La triade d'Héliopolis-Baalbek, Montréal (1985).

HAJJAR Y., « Heliopolitani dei », LIMC 4 (1988), p. 573-592.

HAJJAR Y., « Baalbek, grand centre religieux sous l'Empire (suivi d'un appendice : Supplément II au corpus des documents iconographiques et épigraphiques héliopolitains) », ANRW 2, 18.4 (1990), p. 2458-2508.

Herman D., « Certain Iturean Coins and the Origin of the Heliopolitan Cult », INJ 14 (20002002), p. 84-98.

Herman D., « The Coins of the Itureans », Israel Numismatic Research 1 (2006), p. 51-71. Hill G.F., A Catalogue of Coins in the British Museum. Phoenicia, Londres (1910).

KIndLer A., " On the Coins of the Ituraeans », Actes $d u$ XI ${ }^{e}$ Congrès International de Numismatique 1 (éd. T. Hackens et G. Moucharte), Louvain (1993), p. 283-288.

Lichtenberger A., Kulte und Kultur der Dekapolis, Wiesbaden (2003).

Lightfoot J.L., Lucian. On the Syrian Goddess, Oxford (2003).

Mılik J.T., « Les papyrus araméens d'Hermoupolis et les cultes syro-phéniciens en Égypte perse », Biblica 48 (1967), p. 546-622.

Milı J.T., Recherches d'épigraphie proche-orientale 1. Dédicaces faites par des dieux (Palmyre, Hatra, Tyr) et des thiases sémitiques à l'époque romaine, Paris (1972).

Mouterde R., « Antiquités de l'Hermon et de la Beqâ‘ », MUSJ 29 (1951-1952), p. 19-89.

Mouterde R., «Cultes antiques de la Cœlésyrie et de l'Hermon (Ma'loula, Ba‘albek, Rahlé) », MUSJ 36 (1959), p. 51-87.

Nordiguian L., Temples de l'époque romaine au Liban, Beyrouth (2005).

Norris F.W., " Antioch-on-the-Orontes as a Religious Center, I. Paganism before Constantine », ANRW 2, 18.4 (1990), p. 2322-2379.

Picard C., « Les frises historiées autour de la cella et devant l'adyton dans le temple de Mercure à Baalbek », Mélanges syriens offerts à Monsieur René Dussaud, Paris (1939), p. 319-343.

PuchsteIN O. et al., « Zweiter Jahresbericht über die Ausgrabungen in Baalbek », JDAI 17 (1902), p. 87-124.

ReY-CoQuais J.-P., « Chronique bibliographique », REA 82 (1980), p. 132-133.

Rey-Coquais J.-P., « Apports d'inscriptions inédites de Syrie et de Phénicie aux listes de divinités ou à la prosopographie de l'Égypte hellénistique ou romaine », Egitto $e$ 
storia antica dall'ellenismo all'età araba (éd. L. Criscuolo et G. GeraCI), Bologne (1989), p. 609-619.

Rey-Coquais J.-P., « Sur quelques divinités de la Syrie antique », Mélanges Pierre Levêque 6 (éd. É. Geny et M.-M. Mactoux), Besançon/Paris (1992), p. 247-260.

Rey-Coquais J.-P., « Qalaat Faqra : un monument du culte impérial dans la montagne libanaise », Topoi 9/2 (1999), p. 629-664.

Rey-Coquais J.-P., Inscriptions grecques et latines de Tyr, Beyrouth (2006).

SAIDAH R., «Chronique », BMB 20 (1967), p. 155-180.

SAlamé-SArkis H., « Heliopolitana monumenta », Berytus 35 (1987), p. 126-139.

SChlumberger D., « Le temple de Mercure à Baalbek Héliopolis », BMB 3 (1939), p. 25-36.

SEYrig H., « La triade héliopolitaine et les temples de Baalbek », Syria 10 (1929), p. 314356 (Scripta varia, p. 3-50).

SEYrig H., « Antiquités syriennes. 2. Notes épigraphiques », Syria 12 (1931), p. 318-323 (Antiquités syriennes 1, p. 3-8).

SEYrig H., Antiquités syriennes, Paris (1934-1966).

SEYrig H., «Heliopolitana », BMB 1 (1937), p. 77-100 (Scripta varia, p. 65-89).

SEYRIG H., «Antiquités syriennes. 30. Inscriptions », Syria 20 (1939), p. 302-323 (Antiquités syriennes 3, p. 33-54).

SeYrig H., « Antiquités syriennes. 39. Héraclès-Nergal », Syria 24 (1944-1945), p. 62-80 (Antiquités syriennes 4, p. 1-19).

Seyrig H., "Poids antiques de la Syrie et de la Phénicie sous la domination grecque et romaine », BMB 8 (1949), p. $37-79$ (Scripta varia, p. 367-415).

SeYrig H., « Antiquités syriennes. 55. Le grand prêtre de Dionysos à Byblos », Syria 31 (1954), p. 68-73 (Antiquités syriennes 5, p. 86-91).

SEYrig H., «Antiquités syriennes. 57. Questions héliopolitaines », Syria 31 (1954), p. 80-98 (Antiquités syriennes 5, p. 99-117).

SEYrig H., « Bas-relief de la triade de Baalbek trouvé à Fneidiq », BMB 12 (1955), p. 25-28 (Scripta varia, p. 91-95).

SeYrig H., «Antiquités syriennes. 78. Les dieux de Hiérapolis », Syria 37 (1960), p. 233252 (Antiquités syriennes 6, p. 79-98).

Seyrig H., « Antiquités syriennes. 95. Le culte du Soleil en Syrie à l'époque romaine », Syria 48 (1971), p. 337-373.

SEYrig H., «Antiquités syriennes. 98. Bas-relief des dieux de Hiérapolis », Syria 49 (1972), p. 104-108.

SeYrig H., Scripta varia, Paris (1985).

SIEBERT G., « Hermes », LIMC 5 (1990), p. 285-387.

Sourdel D., Les cultes du Hauran à l'époque romaine, Paris (1952).

Stucky R.A., Die Skulpturen aus dem Eschmun-Heiligtum bei Sidon, Bâle (1993).

Thiersch H., « $\mathrm{Zu}$ den Tempeln und zur Basilika von Baalbek », Nachrichten von den königlichen Gesellschaft der Wissenschaften zu Göttingen, philologisch-historische Klasse (1925), p. 1-24.

Turcan R., « César et Dionysos », Hommage à la mémoire de Jérôme Carcopino, Paris (1977), p. 317-325. 
VAn ANDRINGa W., La religion en Gaule romaine, Paris (2002).

VAN Ess M., K. RHEIDT et al., « Archaeological Research in Baalbek. A preliminary report on the 2004 and 2005 seasons », BAAL 9 (2005), p. 117-146.

VAN Ess M. et T. WeBER, Baalbek, Mayence (1999).

WachteR R., « Die griechischen Inschriften », Das Eschmun-Heiligtum von Sidon. Architektur und Inschriften (éd. R.A. SтUскY), Bâle (2005), p. 319-331.

W Addington W.-H., Inscriptions grecques et latines de la Syrie, Paris (1870).

Wiegand T. (éd.), Baalbek, Berlin/Leipzig (1921-1925).

ZAYAdINE F., « Hermes/Al-Kutbay », LIMC Suppl. (1997), p. 616-619. 


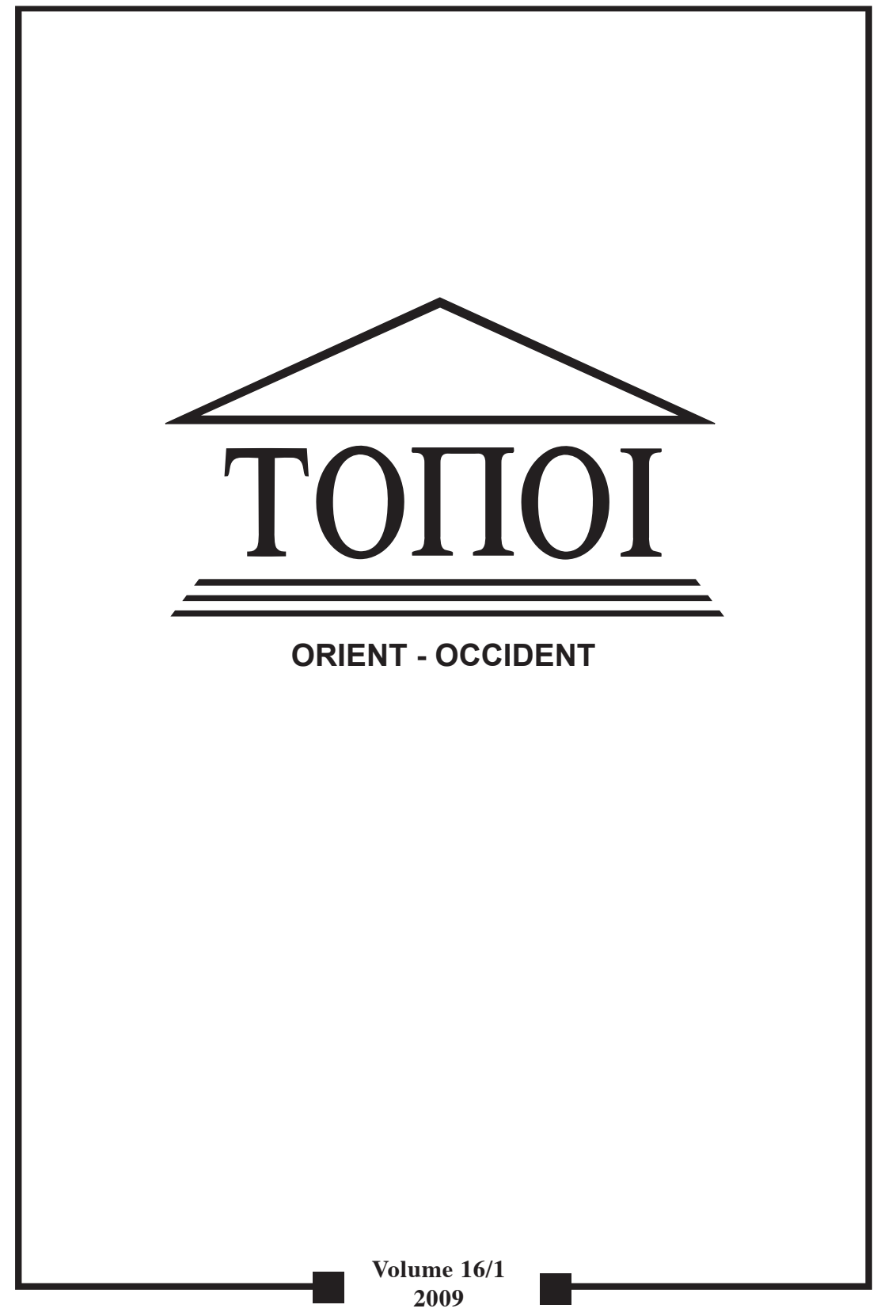



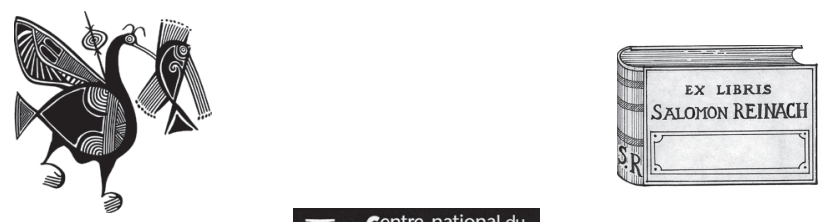
Livre

Ouvrage publié avec le concours

de la Société des Amis de la Bibliothèque Salomon Reinach

et du Centre national du livre

Comité de Rédaction (au 01.01.2009) :

Jean Andreau, Marie-Françoise Boussac, Roland Étienne, Alexandre Farnoux, Ian Morris, Georges Rougemont, Jean-François SAlles, Catherine Virlouvet

Responsable de la Rédaction : Marie-Françoise BousSAC

Adjoint : Jean-Baptiste YoN

Maison de l'Orient Méditerranéen - Jean Pouilloux

7 rue Raulin, F-69365 LYON

Marie-Francoise.Boussac@mom.fr

www.topoi.mom.fr

Traitement de l'image: Anne Flammin (Histoire et Sources des Mondes Antiques)

Diffusion : De Boccard Édition-Diffusion, 11 rue de Médicis, 75006 PARIS

Topoi. Orient-Occident 16, Lyon (2009)

ISSN : 1161-9473

Illustration de couverture : Carte de l'Océan Indien...,Arabie, Inde ; atlas portugais, dit «Atlas Miller» (1519), image BNF, cote GE DD 683 RES. 


\section{SOMMAIRE}

\section{Fascicule 1}

Sommaire 3

Index des auteurs $\quad 5-6$

\section{Dossiers et chroniques}

\section{Recherches sur le monde hellénistique}

A. Magnetro, « La querelle territoriale entre Samos et Priène : $\begin{array}{ll}\text { propositions pour un débat » } & 7-17\end{array}$

P.P. Iossif et C.C. Lorber, « The cult of Helios in the Seleucid East» 19-42

\section{Temples et sanctuaires du Proche-Orient hellénistique et romain}

J. SeIGNE, «La dédicace (?) du sanctuaire d'Artémis de Gérasa de la Décapole. Quelques remarques sur les inscriptions Welles 41 et 109 »

Temples et sanctuaires du Liban (Beyrouth, 26-27 avril 2007)

(édité par P.-L. Gatier, L. Nordiguian et J.-B. Yon)

Introduction

I. PÉRISSÉ-V ALÉRO, « Le sanctuaire romain de Chhîm. Évolution et mutations d'un site cultuel de la montagne libanaise »

T. WALISZEWSKI, « Du temple païen à la basilique chrétienne à Chhîm (Liban Sud) Évolution tardive du sanctuaire (fin $\mathrm{III}^{\mathrm{e}}-\mathrm{VIII}^{\mathrm{e}}$ siècles apr. J.-C.) »

G. Charpentier, «Le petit temple romain du sanctuaire de Yanouh»

J. YASMINE, «Transformations monumentales de sanctuaires et de temples antiques. Les cas de Niha et Hardine»

Z. FANI, « Le tympan ouest et les couronnes du temple A de Niha »

K. Butcher, «Acolytes and Aspergilla : on five coin types of Heliopolis »

J.-B. Yon, « Les inscriptions de Hosn Sfiré »

Y. DABbour et L. TholbeCQ, « Le sanctuaire de Baetocaecé

(Hosn Suleiman, Jabal al-Saheliyé, Syrie) : un état des lieux »

J.P. Rey-CoQuaIs, « Divinités féminines du Liban »

J. AliQuot, « Mercure au Liban »

\section{Proche-Orient antique}

P. Clauss-Balty, « Le mausolée-tour d'Eusébios et Antoninos

à Hass (Syrie du Nord) » 



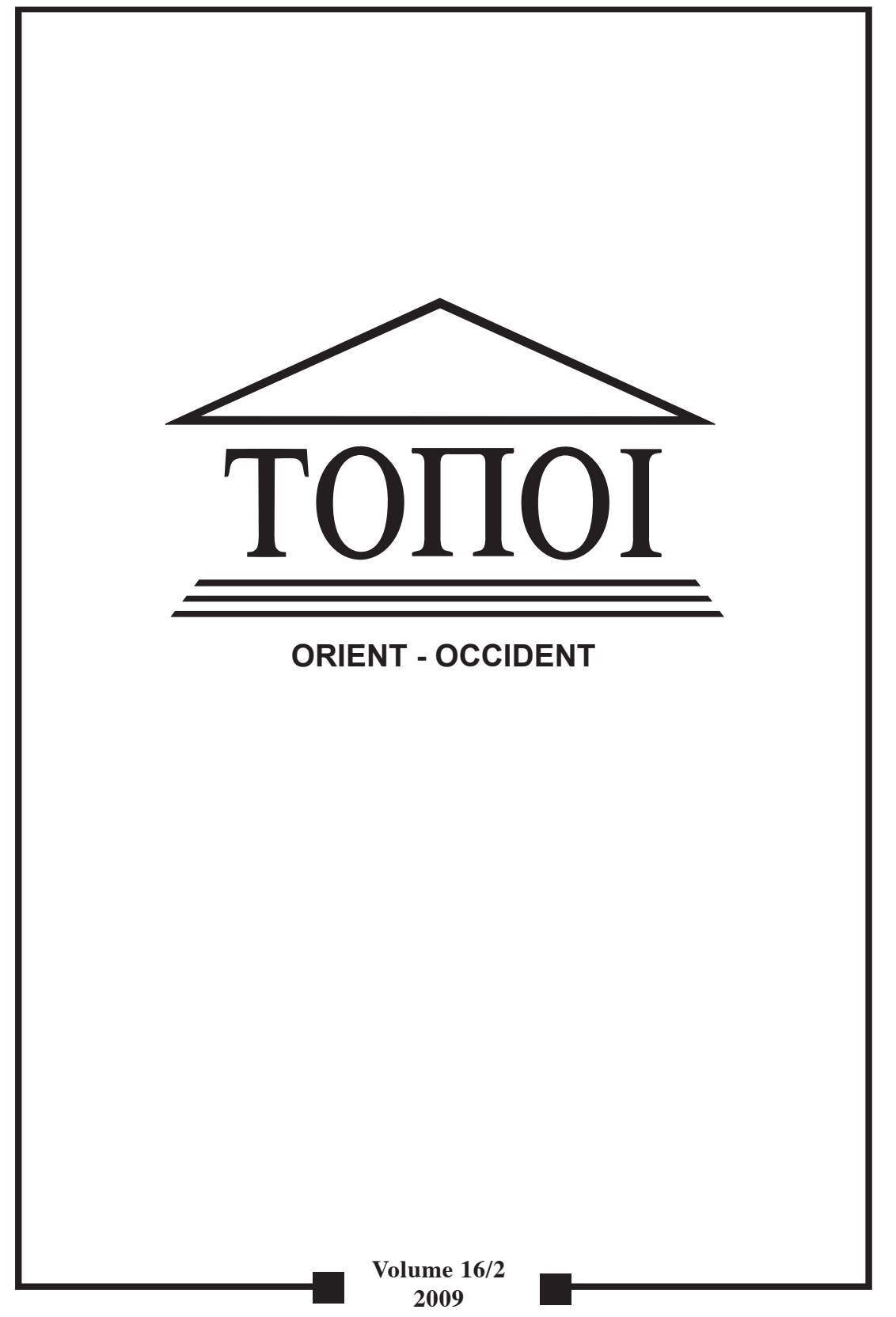



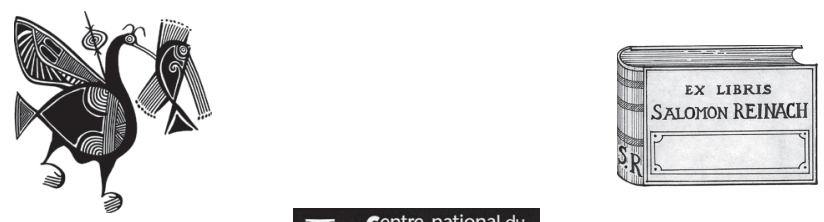
Livre

Ouvrage publié avec le concours

de la Société des Amis de la Bibliothèque Salomon Reinach

et du Centre national du livre

Comité de Rédaction (au 01.01.2009) :

Jean Andreau, Marie-Françoise Boussac, Roland Étienne, Alexandre Farnoux, Ian Morris, Georges Rougemont, Jean-François SAlles, Catherine Virlouvet

Responsable de la Rédaction : Marie-Françoise BousSAC

Adjoint : Jean-Baptiste YoN

Maison de l'Orient Méditerranéen - Jean Pouilloux

7 rue Raulin, F-69365 LYON

Marie-Francoise.Boussac@mom.fr

www.topoi.mom.fr

Traitement de l'image: Anne Flammin (Histoire et Sources des Mondes Antiques)

Diffusion : De Boccard Édition-Diffusion, 11 rue de Médicis, 75006 PARIS

Topoi. Orient-Occident 16, Lyon (2009)

ISSN : 1161-9473

Illustration de couverture : Carte de l'Océan Indien...,Arabie, Inde ; atlas portugais, dit «Atlas Miller» (1519), image BNF, cote GE DD 683 RES. 


\section{SOMMAIRE}

\section{Fascicule 2}

Sommaire

335-336

Chronique : Achéménides

R. BOUCHARLAT, «Quelques ouvrages sur l'art et l'archéologie achéménides »

337-346

Chronique : Cités grecques à l'époque hellénistique

P. HAMON, «Démocraties grecques après Alexandre. À propos de trois ouvrages récents »

$347-382$

Chronique : Épigrammes et collections

G. SAuron, « À propos de Évelyne Prioux, Petits musées en vers. Épigramme et discours sur les collections antiques (2008)»

\section{Comptes rendus d'ouvrages}

S. Amigues, L. Bodiou, D. Frère et V. Mehl (dir.), Parfums et odeurs dans l'Antiquité (2008) ; A. Verbanck-Piérard, N. Massar, D. Frère (éds), Parfums de l'Antiquité. La rose et l'encens en Méditerranée (2008)

$395-420$

S. Amigues, A. Durand (éd.), Plantes exploitées, plantes cultivées. Cultures, techniques et discours (2007)

P. BRulé, St. Georgoudi, R. Koch Piettre et Fr. Schmidt (éds), La cuisine et l'autel. Les sacrifices en questions (2005)

V. DAsen, A. Cohen et J.B. Rutter (éds), Constructions of Childhood in Ancient Greece and Italy, Hesperia Supplement 41 (2007)

R. ÉtIENnE, R. Descat (éd.), Approches de l'économie hellénistique (2006)

Fr. QueYrel, P. Schultz, R. von den Hoff (éds), Early Hellenistic Portraiture Image, Style, Context (2007)

Fr. de Callataÿ, K. Dahmen, The Legend of Alexander the Great on Greek and Roman Coins (2007)

$465-468$

M. SARTRE, S. Follet (éd.), L'hellénisme d'époque romaine. Nouveaux documents, nouvelles approches (2004)

N. Laubry, J. Scheid (éd.), Pour une archéologie du rite. Nouvelles perspectives de l'archéologie funéraire (2008)

V. Gasparini, M. Malaise, Pour une terminologie et une analyse des cultes isiaques (2005)

R. ÉtIenne, Fr. QueYrel et B. Redon, M. Trümper, Die 'Agora des Italiens' in Delos (2008)

J.-Ch. Moretti, M.C. Sturgeon, Corinth IX, iii, Sculpture : the Assemblage from the Theater (2004) 
J.-Ch. Moretti, Fr. Sear, Roman Theatres. An Architectural Study (2006)

D. Keller, B. Czurda-Ruth, Hanghaus 1 in Ephesos. Die Gläser (2007)

\section{Iran, Asie centrale}

R. Boucharlat, E. Errington and V. Sarkhosh Curtis, From Persepolis to the Punjab. Exploring ancient Iran, Afghanistan and Pakistan (2007)

R. Boucharlat, P. Callieri, L'archéologie du Fārs à l'époque hellénistique. Quatre leçons au Collège de France (2008)

Ch. Baratin, Ch. Lerouge, L'image des Parthes dans le monde gréco-romain. Du début du rer siècle av. J.-C. jusqu'à la fin du Haut-Empire romain (2007)

L. Martinez-SÈve, J. Cribb, G. Herrmann (éds), After Alexander. Central Asia before Islam, Proceedings of the British Academy 133 (2007)

\section{Proche-Orient, Chypre}

A. Cannavò, Hommage à Annie Caubet, Cahiers du Centre d'Études Chypriotes 37 (2007)

K. Butcher, G.M. Cohen, The Hellenistic Settlements in Syria, the Red Sea Basin, and North Africa (2006)

O.D. Hoover, L. Capdetrey, Le pouvoir séleucide. Territoire, administration, finances d'un royaume hellénistique (2007)

Fr. de Callataÿ, O.D. Hoover, Coins of the Seleucid Empire from the Collection of Arthur Houghton. Part II (2007)

J.-B. Yon, R.A. Stucky et alii,, Das Eschmun-Heiligtum von Sidon : Architektur und Inschriften, Antike Kunst Beiheft 19 (2005)

J.-B. Yon, T. Kaizer (éd.), The Variety of Local Religious Life in the Near East in the Hellenistic and Roman Periods (2008)

G. Bowersock, J. Aliquot, IGLS XI. Mont Hermon (Liban et Syrie) (2009)

Égypte

D. Rathbone, C. Adams, Land Transport in Roman Egypt. A Study of Economics and Administration in a Roman Province (2007)

M. Drew BEAR, Laurens E. Tacoma, Fragile Hierarchies, The Urban Elites of Third-Century Roman Egypt (2006)

R. Bagnall, J. Gascou, Fiscalité et société en Égypte byzantine (2008)

G. RuFFINI, P. Sarris, Economy and Society in the Age of Justinian (2006)

\section{Méditerranée, Mer rouge, Golfe et Océan indien}

F. De Romanis, « Zavorra e commercio 'triangolare' nell'oceano Indiano : a proposito di un libro recente »

F. De Romanis, R.T.J. Cappers, Roman Foodprints at Berenike. Archaeobotanical Evidence of Subsistence and Trade... (2006)

F. De Romanis, H. Cuvigny, Ostraca de Krokodilô. O. Krok. 1-151. Praesidia du désert de Bérénice II (2005)

F. De Romanis, D. Peacock / L. Blue (eds.), Myos Hormos-Quseir al-Qadim. Roman and Islamic Ports on the Red Sea. Volume 1 (2006)

M. TuChSCHERER, R.E. Margariti, Aden and the Indian Ocean Trade. 150 Years in the Life of a Medieval Arabian Port \& (2007)

É. VALLET, S.D. Goitein et M.A. Friedman, India Traders of the Middle Ages : Documents from the Cairo Geniza «India Book» (2008) 